\title{
La Belgique dans les épisodes de séries françaises coproduits avec la RTBF : de l'Atomium au conflit linguistique
}

\author{
Sarah Sepulchre \\ Université catholique de Louvain
}

Les récits populaires véhiculent des représentations ${ }^{1}$, que certains considèrent comme figées. Umberto Eco analysant le personnage de James Bond considère que Fleming a recours « aux attraits les plus universels et les plus sûrs et met en jeu des éléments archétypiques » (Eco 90). Les séries télévisées n'échappent pas à la règle. Cela permet de les analyser sous un angle sociologique et de vérifier ce qu'elles disent du monde ${ }^{2}$. Dans ce numéro consacré aux Francophones, il nous semblait intéressant de vérifier le portrait qui en est produit dans les fictions plurielles. Comme nous sommes belges, le traitement réservé aux Belges nous intéressait particulièrement. Il existe peu de séries belges francophones et peu de fictions étrangères mettant en scène les Belges, ce qui avait l'avantage de circonscrire un corpus manipulable. Isabelle Veyrat-Masson remarque, dans un article sur les stéréotypes nationaux à la télévision (1989 243-244), que les publics français, américains et japonais interrogés s'accordent sur les images construites à la fois sur les autres et, c'est plus étonnant, sur euxmêmes. Cependant, s'ils acceptent les stéréotypes sur eux-mêmes s'ils sont véhiculés par leur propre télévision, ils les rejettent s'ils sont énoncés par les médias étrangers. Nous avons donc décidé de travailler sur l'image des Belges véhiculée par des séries françaises en partant du principe que nous serions plus capable de les repérer et de les analyser ${ }^{3}$.

\section{Les représentations sociales}

1 Dans cet article, nous utiliserons les concepts de "représentation sociale" et de "stéréotype" comme des synonymes. Comme Jean-Louis Dufays (1994) ou Ruth Amossy (1991) nous considérons que les différences entre les deux sont des variations de figements des images et non une différence de nature.

2 Dominique Pasquier dans La culture des sentiments, Sabine Chalvon-Demersay et ses recherches sur Urgences ou les adaptations, Eric Macé dans La société et son double, pour ne citer qu'eux, ont produit des analyses convaincantes des représentations présentées dans les récits.

3 Par ailleurs, une analyse des représentations véhiculées par les séries belges francophones a déjà fait l'objet d'un article : Sarah Sepulchre, "Melting Pot : An ambiguous series combining minority and majority discourses" dans Alternative francophone (vol. 1, n 6, 2013). 
«La fiction a, fondamentalement, un effet de désindividualisation : ce en quoi, d'ailleurs, elle concerne très directement les sciences sociales, en particulier la sociologie et l'anthropologie » (Heinich 70). Il nous semble, en effet, que les séries télévisées peuvent être considérées comme des acteurs sociaux, partageant des représentations sociales. Jean-Pierre Esquenazi, discutant des propositions émises par Eric Macé, constate que les programmes de télévision ont une double nature : ils sont produits par un contexte et ils sont des discours. "L'objet lui-même, de par sa double nature, est le point d'articulation de la sociologie des médias : produit, il est le résultat des processus sociaux [de production et de réception] ; discours, il en donne une représentation.»(94) L'auteur pose que "la télévision représente un accompagnement "réaliste" de la réalité sociale » (90).

Si on rapproche deux textes composés par Sabine Chalvon-Demersay, on trouve une démonstration proche de celle de Jean-Pierre Esquenazi. L'auteure analysant les adaptations du Bossu à la télévision française montre que les scénaristes ne sont pas déconnectés du monde auquel ils appartiennent. Au contraire, ils transforment le récit initial en fonction de ce qui est vraisemblable et acceptable au moment où ils écrivent (ChalvonDemersay 2005/3 81). Dans une recherche plus ancienne, cette même auteure avait analysé les scénarios reçus par France 2 et montrait qu'ils étaient porteurs d'un certain air du temps (Chalvon-Demersay 1994). Autrement dit, les fictions télévisuelles ne sont pas isolées de la société qui leur est contemporaine. Elles ne sont certainement pas des reflets exacts, mais la télévision étant composée d'individus insérés dans le monde, elle participe aux représentations circulant à un moment donné.

Cette interpénétration des discours et des acteurs sociaux est d'ailleurs constitutive de la définition concoctée par Serge Moscovici. Ce dernier considère que, si ces représentations sont sociales, c'est d'abord parce qu'elles sont «socialement partagées ». Elles font consensus dans le groupe. Deuxièmement, le processus qui les crée est basé sur l'interaction : « en se représentant une chose ou une notion, on ne se fait pas uniquement ses propres idées et images. On génère et transmet un produit progressivement élaboré dans d'innombrables lieux selon des règles variées. Dans ces limites, le phénomène peut être dénommé représentation sociale. » (Moscovici 83).

Les représentations ne sont pas élaborées par un individu, mais pas une multitude d'individus qui les énoncent et les transmettent constamment. On ne trouve donc pas un individu-origine, un lieu-origine ou un momentorigine de la représentation sociale, mais une création diffuse. La représentation est sociale parce qu'elle doit être créée innombrablement et parce qu'elle doit être transmise au sein du groupe.

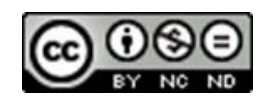


Denise Jodelet, une autre scientifique qui a travaillé cette question, précise quelques caractéristiques de ces représentations sociales. Elles sont socialement élaborées et partagées ( $c f$. supra), elles sont des connaissances naïves (elles sont spontanées et s'opposent en cela aux savoirs scientifiques), elles sont des connaissances pratiques (elles permettent de conceptualiser la réalité, de la comprendre, d'expliquer les faits, elles servent de guides pour l'action), elles sont consensuelles (elles ont été négociées au sein du groupe et deviennent des évidences), elles sont des schématisations qui représentent l'objet caractérisé (elles sont donc réductrices) (Jodelet 366-367).

Les représentations ont donc des fonctions précises au sein des sociétés. Elles permettent la cohésion du groupe. Elles sont des guides de vie pour les individus. Elles doivent donc être crédibles. François Flahaut note que les représentations, reçues comme des témoignages véridiques, sont considérées comme de l'information (Flahaut 37). Mais il souligne aussitôt l'ambiguïté du processus de reconnaissance de la véridicité des images véhiculées. En effet, la plupart du temps, l'humain ne confronte pas les représentations avec la réalité factuelle. «Le plus souvent en effet, nous considérons comme véridiques les représentations qui sont réputées l'être par le plus grand nombre de ceux qui nous entourent ou par des instances qui font autorité. » (Flahaut 38) C'est ce qu'il nomme des croyances. Cela se complique encore quand l'auteur constate que la plupart des éléments contenus dans les représentations ne sont jamais explicitement évalués. Prenant l'exemple d'un film historique, il souligne que les «traits saillants du récit (événements décisifs, actions et réactions des personnages principaux) » sont commentés, examinés par les récepteurs. Par contre, les éléments « défocalisés et qui, par conséquent, restent dans la pénombre de notre conscience implantent en nous des images sur le statut desquelles nous ne nous interrogeons guère »(Flahaut 45). Les représentations partagées, les évidences de bon sens n'attirent donc pas l'attention critique. "Disons [...], que les croyances auxquelles on adhère consciemment [...] doivent être distinguées de celles auxquelles on adhère sans le savoir. Celles-ci que, faute d'un meilleur terme, j'appelle des mi-croyances, présentent sans doute un large éventail. » (Flahaut 46) Ces mi-croyances, par leur nature même, sont donc difficilement contestables. Jean Pirotte avance même que le stéréotype appartient à un fond culturel «non critiqué et irrationnel » (Pirotte 1).

À cette ambiguiité des représentations s'ajoute une autre caractéristique, qui est généralement la plus critiquée, il s'agit de la simplification. Denise Jodelet le soulignait déjà, les représentations sont des schématisations. Dans un article concernant l'image donnée de Marseille dans Plus belle la vie, Céline Bryon-Portet note que la ville est présente à l'image :

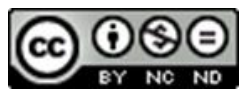




\begin{abstract}
Si nombre de ses scènes se déroulent à proximité de lieux et de monuments symboliques de la cité phocéenne, la série immerge également le téléspectateur dans les habitus de ses habitants. Patrimoine architectural (Notre-Dame de la garde, le Vieux port, la Gare Saint-Charles, la prison des Baumettes...), patrimoine culturel et social (la bouillabaisse et le pastis, le célèbre accent marseillais, la plage du Prado, le Parc Borély), sont ainsi régulièrement sollicités. Certains personnages principaux incarnent d'ailleurs, à eux seuls, cette culture marseillaise : Roland Marci, par exemple [...] («Les productions télévisées » \$6)
\end{abstract}

Cependant, elle s'interroge directement. La série ne véhicule-t-elle pas une image réductrice de Marseille. "Car au-delà de cette large diffusion, qui permet de faire connaitre ou d'accroître la visibilité de la ville, Plus belle la vie livre une représentation de la cité phocéenne réduite à ses symboles les plus populaires.» («Les productions télévisées» \$20) Les représentations sociales sont donc ambivalentes : à la fois nécessaires à la société, salutaire pour nos esprits humains incapables de gérer trop d'informations; à la fois parcellaires (ouvrant donc la porte à l'erreur ou la discrimination) et peu contestées (car devenues évidentes). Céline Bryon-Porter, elle-même, constatait dans l'article que le patrimoine de Marseille, même s'il n'est présent que de manière partielle, permet de donner une certaine crédibilité à la fiction. Elle établissait alors un parallèle avec la fonction spéculaire de l'image « [...] tout à la fois mimétique [...] du réel puisqu'elle entretient des rapports analogiques avec ce dernier, et double déformé qui nous plonge dans l'univers du rêve (Morin, 1965), mais qui participe pourtant de la construction de la réalité. » («Les productions télévisées» \$22) Et elle ajoutait que cette bipolarité a également été soulignée par des géographes "pour lesquels l'image de la ville est scindée entre la ville réelle et la ville des représentations mentales $[. .] ».((«$ Les productions télévisées » \$22).

\title{
2. Les stéréotypes nationaux
}

Parmi les représentations que nous manipulons tous les jours, certaines ont trait à des territoires, des nations ou leurs habitants. Elles nous intéressent au premier chef dans le cadre de cet article centré sur l'image qui est donnée de la Belgique et des Belges dans les séries françaises. Sylvaine Marandon considère que ces stéréotypes proviennent des rapports économiques entre deux pays, des récits de voyages, d'échanges culturels comme les tournées théâtrales, des romans, des manuels d'éducation (Pirotte 7-8). François Crouzet ajoute à ces sources la tradition orale au sein du milieu familial, les voyages, la presse, le cinéma et la télévision (Crouzet 132-133). Ces stéréotypes « reposent sur le postulat que tous les membres d'une nation présentent des traits de caractère communs et immuables » (Crouzet 134).

En effet, Joseph Fichter considère que les stéréotypes sont constitués par deux processus. Le premier est la généralisation: "c'est le procédé 
mental par lequel nous appliquons le fait connu au sujet d'une personne ou de quelques personnes à toutes celles qui appartiennent à la même catégorie ». Le second est la spécification: «c'est le procédé inverse, par lequel nous appliquons à une personne individuelle le stéréotype que nous avons en tête au sujet de la catégorie entière à laquelle elle appartient » (Tilleux-Craenhals 127-128).

Donc ces stéréotypes nationaux, comme toutes représentations, ne sont pas contestés ( $c f$. supra) et ils sont obtenus par amplification ou simplification d'éléments. Comme toute image, leur véracité peut être mise en doute. Et pourtant, ils ont des conséquences bien réelles. Jean Pirotte (1) souligne qu'ils jouent un rôle certain dans les relations entre les peuples. François Flahaut considère que les croyances peuvent modifier la réalité vécue par un groupe ou par les citoyens d'un État (Flahaut 41). Une conséquence étonnante relevée par Isabelle Veyrat-Masson est que les individus ont tendance à se conformer aux stéréotypes véhiculés à leur encontre. Nous l'avons souligné au début de ce texte, les personnes qu'elle a interrogées reconnaissent toutes les mêmes stéréotypes qu'ils concernent les autres ou soi-même. Se produit alors un cercle vicieux puisque les personnes produisent les comportements qu'on attend d'eux. «Le stéréotype national c'est une réputation, bonne ou mauvaise, il est difficile de s'en défaire, bonne ou mauvaise, chacun a tendance à s'y conformer » (Veyrat-Masson 244). Les stéréotypes, rappelle-t-elle, sont antérieurs à l'expérience que nous faisons du monde, «l'enfant [les] apprend "au biberon" »(248). Une fois acquis dans le processus de socialisation, ils filtrent et orientent notre expérience. "Les représentations deviennent alors la réalité, on ne fait plus de différences entre les expériences directes et les informations reçues à travers des intermédiaires, des médias (d'où la difficulté à repérer l'influence des médias). »(Veyrat-Masson 248) Même quand l'être humain voyage, il confirme ce qu'il attendait en vertu du phénomène de perception sélective. «[...] les individus sélectionnent dans le réel ce qui confirme leurs croyances préalables. » (248).

Les stéréotypes nationaux manifestent une grande stabilité (Pirotte 1); cela ne signifie cependant pas qu'ils n'évoluent pas. En effet, les opinions que les groupes portent les uns sur les autres varient en fonction de leur interaction. Les relations entre pays sont tributaires de leurs rapports de forces (Veyrat-Masson 250). François Crouzet s’intéressant aux images des Français et des Britanniques véhiculées entre les deux pays montre qu'elles sont sujettes à variation en fonction des relations diplomatiques entre les pays. Par exemple, la croyance en l'hypocrisie des Britanniques s'est développée à la suite de la bataille de Waterloo quand Wellington a fait enlever des œuvres d'art du Louvre. Elle était alors une idée très répandue concentrée dans l'expression «La Perfide Albion ». L'idée est pratiquement abandonnée après la Seconde Guerre mondiale, mais ressurgit 
implicitement dans les critiques de la politique britannique sur les questions européennes. Un autre stéréotype a lui complètement disparu, c'était l'idée que les Britanniques s'ennuyaient, avaient une tendance à la dépression, au « spleen ». Cette vision n'a pas résisté au Swinging London des années 1960. (Crouzet 135)

Par ailleurs, les stéréotypes ne sont pas des objets figés, mais naissent dans les interactions. Thomas Glesener décortique comment les images du Flamand ivrogne et de la Flamande aux mœurs dissolues se sont développées dans la société espagnole du XVIe siècle. Il constate que ces stéréotypes doivent être analysés dans les relations entre les deux peuples. Ils révèlent une volonté de comprendre un mode de vie flamand de la part des Espagnols, une tentative d'intelligibilité des situations, une tentative de transmettre une expérience en retour de voyage. Ces clichés ne peuvent être détachés du contexte de l'époque et des acteurs qui les ont forgés, ainsi que de leurs caractéristiques (Glesener 337-350).

Enfin, il n'existe pas un stéréotype national à un moment donné, mais plusieurs couches de représentations «depuis la vue des experts ou spécialistes qui se sont consacrés à l'étude de ce pays jusqu'aux stéréotypes élémentaires, aux formules toutes faites [...]»(Crouzet 132) Au point qu'au sein d'une même communauté, des images très différentes peuvent coexister en fonction des milieux sociaux et des tendances politiques. «De même pour ce qui est de la nuance affective - admiration, sympathie, ou bien hostilité, mépris - qui s'y ajoute fréquemment, créant dans l'opinion de chaque pays une division entre "philes" et "phobes". » (Crouzet 137)

Les stéréotypes sur les Belges sont légions et souvent bien connus. À propos de la bande dessinée, Ghislaine Tilleux-Craenhals repère des stéréotypes positifs (générosité, bonhomie accueillante, flatterie) et des clichés plus négatifs (le Belge est rouspéteur) attachés au personnage de monsieur Lambique dans Bob et Bobette (118). Dans l'œuvre d'Hergé, Tintin est une figure plus internationale (plus vendeur à l'étranger) et les personnages principaux sont francisés (Haddock lit le Paris-Flash). Cependant, les traits typiques reviennent dans un personnage plus secondaire comme monsieur Lampion. Il est d'abord vu comme peu stylé, pourvu d'un sens de l'humour assez plat, sans-gêne et rouspéteur. Au fur et à mesure de l'histoire, il fait évoluer l'intrigue bien malgré lui et devient alors jovial, de bonne humeur et bon vivant (Tilleux-Craenhals 127-128). Les frites, le chocolat, l'accent belge, le Wallon fan de foot et paresseux, le Flamand bilingue et ambitieux, la capacité d'autodérision ou de compromis, le Belge ayant une brique dans le ventre, fêtard, raciste sont 
parmi les caractéristiques les plus souvent citées ${ }^{4}$. Cependant, nous ne souhaitons pas ici établir une liste précise de ces idées reçues que nous comparerions ensuite aux séries analysées. Notre démarche sera plus inductive (cf. infra).

\section{Stéréotypes et télévision}

La plupart des auteurs s'intéressant au stéréotype dans la fiction constatent que son utilisation est inévitable. C'est le cas si le personnage à croquer, par exemple, est un personnage secondaire un peu grossier. Sylvaine Marandon, analysant l'image des Français et des Juifs dans la littérature anglaise, remarque que les romans proposent des portraits, des tableaux de genre mettant en scène des étrangers. "Ceux-ci ne peuvent qu'être, en principe, stéréotypés, puisque telle est généralement leur fonction: fournir des personnages secondaires pittoresques, aisément reconnaissables, suggérant automatiquement un certain comportement lié à leur identité ethnique.» (Marandon 8), Mais au-delà de ces personnages secondaires voués à faire rire ou à jouer les victimes, le recours aux stéréotypes est une nécessité à tous les niveaux d'écriture: de la construction simple des phrases, car le langage est une convention, au choix des scénarios types, à la manière dont les lecteurs donnent sens à ce qu'ils lisent en activant également des schémas types. C'est la thèse de JeanLouis Dufays tout au long de son ouvrage Stéréotype et lecture. L'utilisation des stéréotypes dans la fiction télévisuelle parait encore plus " naturelle ». Isabelle Veyrat-Masson souligne les analogies entre les deux.

Les deux fonctionnent sur la répétition à des millions d'exemplaires (stéréomass) d'assertions (type); la télévision comme le stéréotype, ressemble à tel point au réel qu'ils deviennent la réalité alors qu'ils n'en sont qu'une représentation. Tous deux sont des montages d'images. Ce sont également des instruments de communication qui ont comme «vocation » de rendre accessible et simple ce qui est compliqué et surtout ce qui est multiple donc différencié. Enfin leur objectif est de rendre compte de l'abstrait. (240)

Plus encore, Isabelle Veyrat-Masson considère que la télévision produit des stéréotypes consciemment (" pour faire rire, pour faire vite, pour faire sens », 245), mais qu'elle en crée également malgré elle. "Mais surtout, il nous est apparu que même lorsque la télévision déclare vouloir lutter contre, elle ne réussit qu’à propager d'autres stéréotypes. » (245)

\footnotetext{
${ }^{4}$ Lire par exemple : http://europeisnotdead.com/europe-is-not-deadfr/video/imageseuropeennes/stereotypes-europeens/

$\mathrm{Ou}$

http://www.uclouvain.be/cps/ucl/doc/spri/documents/Article Min Reuchamps Preju ges.pdf
} 
Cependant les auteurs s'accordent pour affirmer que l'auteur de la fiction n'est pas le créateur du stéréotype. «On peut tenir pour acquis que l'écrivain exprime en partie quelque chose d'existant - ici, une image, qu'il a héritée, même si c'est inconsciemment, de son expérience et de son milieu » (Marandon 12-13). Isabelle Veyrat-Masson établit que les stéréotypes découverts existaient bien avant que la télévision n'ait été inventée (242). Ce qui n'exonère pas totalement les auteurs de fiction de toute responsabilité. En effet, il est également largement attesté que les créateurs ont un "rôle amplificateur certain » (Marandon 12-13) et que les stéréotypes sont renforcés par la télévision (Veyrat-Masson 242). Henri Boyer le constate également, le stéréotype est «communicationnellement rentable». C'est ce qui explique que «les médias en font un usage immodéré ». Ainsi, si «les médias ne créent pas de toutes pièces les stéréotypes, ils contribuent largement au processus de stéréotypage » (\$10). Cette «responsabilité» des médias et de la fiction vis-à-vis des représentations est encore accentuée par le fait qu'ils sont aujourd'hui, les «principaux pourvoyeurs [de matière représentationnelle] » (Boyer \$2). Ce qui vaut aussi pour les pays et leurs citoyens. Isabelle Veyrat-Masson souligne que les mille étudiants interrogés ont peu voyagé et que la télévision est leur principale source d'information sur les étrangers (241).

Concernant les stéréotypes nationaux, un autre élément lié à la structuration du paysage audiovisuel intervient : les nations. La télévision est soutenue financièrement par les pouvoirs publics « en raison de l'adage selon lequel les biens culturels ne sont pas des biens comme les autres » (Dagnaud 35). «[...] leur impact sur la formation des goûts et de l'opinion [...] justifie des politiques de régulation et de subventions que pratiquent tous les grands pays [...]» (Dagnaud 36). Le territoire d'activité d'un média audiovisuel est donc d'abord national. "On oublie combien les médias œuvrent sur une matrice identitaire nationale [...] En France, la politique de l'exception culturelle permet de stimuler l'identité nationale par le biais de fictions et documentaires qui parlent à la société d'elle-même.» (Dagnaud 37)

Les programmes sont porteurs d'un «imaginaire national» (Dagnaud 244) pour plusieurs raisons. D'abord, c'est économiquement plus porteur, car cela permet de drainer un public plus large. Cela permet aussi de recevoir des subventions, puisqu'il s'agit d'une injonction politique. Monique Dagnaud parle de projet «quasiment civique», «fortement suggéré par les textes réglementaires» (246). Ces fictions reçoivent de plus les suffrages des téléspectateurs, "ceux-ci se laissant porter par cette représentation vaguement idéalisée et stéréotypée de la société française » (247). "Les fictions en particulier, genre télévisuel de loin le plus onéreux, sont assujetties à cette logique. Leurs commanditaires endossent, pour plaire au public, l'esprit de clocher [...]» (Dagnaud 244). 
Benoît Lafon décortiquant les représentations véhiculées par trois séries diffusées par France 3, soulignait l'importance des lieux de ruralité, des univers campagnards familiers, des personnages proches des classes moyennes, des leitmotive récurrents (les abus de la justice, le poids de l'héritage...) et offrait des conclusions proches de celles de Monique Dagnaud sur la société idéalisée proposée par la fiction française.

Pourtant, ce n'est pas la seule voie possible. Isabelle Veyrat-Masson constate avec surprise que les télévisions françaises et japonaises diffusent peu d'émissions sur les États-Unis alors que ce pays semble omniprésent. Il l'est, en effet, mais à travers sa propre production, spécialement des fictions. " $[\ldots]$ les Américains sont responsables de leurs stéréotypes, toutefois leurs émissions sont si nombreuses, si diverses (de Starsky et Hutch à La petite maison dans la prairie) que la multiplication des stéréotypes en détruit le caractère simplificateur et réducteur pour donner une image assez riche de ce pays $[. .] ».(243)$ Plusieurs avantages peuvent être tirés de cette situation. D’abord, le pays contrôle l'image qui est diffusée de lui-même. Ensuite, il promeut son territoire, mais aussi son mode de vie et ses produits. Monique Dagnaud le signale : «Les productions médiatiques [...] constituent un vecteur efficace pour l'exportation du french way of life, et donc le cortège de produits nationaux qui l'incarne. Les influences culturelles ne sont jamais candides » (39). Enfin, on l'a déjà vu, Isabelle Veyrat-Masson remarque que les téléspectateurs acceptent les stéréotypes s'ils viennent d'un compatriote. La multiplication et la diversité des stéréotypes présents dans les fictions tranchent par contre avec cette uniformisation de l'image idéalisée de la France constatée par Monique Dagnaud. Les fictions américaines proposeraient donc une manière un peu différente de traiter l'image d'un pays et les stéréotypes.

\section{Méthodologie}

Le corpus sélectionné pour cet article est constitué d'épisodes de séries françaises coproduits avec la chaine publique belge francophone, la RTBF. Comme les deux chaînes mettent en commun leur moyen, c'est généralement l'occasion soit d'amener le héros en Belgique, soit de lui faire rencontrer des Belges. Le fait de travailler sur ces épisodes nous garantit pratiquement de récolter des stéréotypes. Cependant, cela ne signifie pas que les Belges soient récurrents dans les séries. En effet, ils restent rares en dehors de ces épisodes et les coproductions restent exceptionnelles. Monique Dagnaud avance qu'en 2003 cette forme de financement a seulement couvert 8,8\% du devis des fictions télévisées (39, note 2).

Depuis les années 1990, trente-sept épisodes de dix-neuf séries ont ainsi été diffusés (voir la liste ci-dessous). Pour cet article, nous avons 
étudié presque toutes les séries concernées. Nous avons exclu Les Steenfort, maîtres de l'orge ${ }^{5}$, Le Destin des Steenfort et Maigret, car ces séries se déroulent dans le passé or ce sont les représentations contemporaines qui nous intéressent. Nous avons également rejeté la série Rose et $V$ al, car, s'il s'agit bien d'une coproduction, l'épisode en question ne se déroule pas en Belgique et ne présente pas de Belges. Quand la fiction connait plus d'un épisode belge, nous en avons sélectionné un au hasard. Le corpus compte donc seize épisodes de seize feuilletons différents.

Le corpus comporte des fictions de tous formats (26, 52 et 90 minutes) et de tous genres (séries et collection de téléfilms). Il s'agit principalement de fictions policières et de séries dramatiques, à l'exception de Plus belle la vie qui est un soap opera.

Tableau 1 : Épisodes de séries françaises coproduits par la RTBF (en grisé le corpus sélectionné pour cet article)

\begin{tabular}{|c|c|c|c|c|}
\hline $\begin{array}{l}\mathbf{N} \\
0\end{array}$ & Séries & $\begin{array}{l}\text { Format et } \\
\text { genre }\end{array}$ & Épisodes & $\begin{array}{l}\text { Date de } \\
\text { diffusion }^{6}\end{array}$ \\
\hline \multirow[t]{5}{*}{1} & \multirow[t]{5}{*}{ Maigret } & \multirow[t]{5}{*}{$\begin{array}{l}90^{7} \\
\text { (série) }\end{array}$} & $\begin{array}{l}\text { Maigret chez les } \\
\text { Flamands }\end{array}$ & $09 / 11 / 1991$ \\
\hline & & & $\begin{array}{l}\text { Les vacances de } \\
\text { Maigret }\end{array}$ & $20 / 05 / 1995$ \\
\hline & & & $\begin{array}{l}\text { Maigret et l'inspecteur } \\
\text { cadavre }\end{array}$ & $04 / 01 / 1998$ \\
\hline & & & $\begin{array}{l}\text { Maigret et la croqueuse } \\
\text { de diamants }\end{array}$ & $21 / 12 / 2000$ \\
\hline & & & $\begin{array}{l}\text { Les scrupules de } \\
\text { Maigret }\end{array}$ & $08 / 05 / 2003$ \\
\hline \multirow[t]{2}{*}{2} & \multirow[t]{2}{*}{ C'est mon histoire } & \multirow{2}{*}{$\begin{array}{l}52 \\
\text { (collection) }\end{array}$} & Femme de mon père & $25 / 05 / 1993$ \\
\hline & & & École d'enfer & $08 / 09 / 1993$ \\
\hline 3 & Nestor Burma & $\begin{array}{l}90 \\
\text { (série) }\end{array}$ & $\begin{array}{l}\text { Un croque-mort } \\
\text { nommé Nestor }\end{array}$ & $27 / 02 / 1993$ \\
\hline 4 & $\begin{array}{l}\text { Les Renseignements } \\
\text { généraux }\end{array}$ & $\begin{array}{l}90 \\
\text { (série) }\end{array}$ & Mortelle amitié & $07 / 05 / 1994$ \\
\hline 5 & Le R.I.F. & $\begin{array}{l}90 \\
\text { (série) }\end{array}$ & $\begin{array}{l}\text { Piège pour enfants } \\
\text { seuls }\end{array}$ & $28 / 02 / 1996$ \\
\hline $6 a$ & $\begin{array}{l}\text { Les Steenfort, maitres } \\
\text { de l'orge }\end{array}$ & $\begin{array}{l}3 \times 90 \\
\text { (minisérie) }\end{array}$ & & $\begin{array}{l}7,14, \\
21 / 11 / 1996\end{array}$ \\
\hline 6 & Le destin des Steenfort & $3 \times 90$ & & 25/05, 01, \\
\hline
\end{tabular}

5 Par ailleurs, Jean Van Hamme, l'auteur de la bande dessinée et de son adaptation, explique que TF1, la chaine privée française qui a diffusé la minisérie, a exigé que l'intrigue soit déplacée en France afin de « s'adapter aux attentes (supposées) des différents publics. » (Blandin 147). Voir aussi Chris Reyns-Chikuma, "Economie et identité" The French Review, 84.2, 2010, 342-57.

6 Les séries apparaissent de manière chronologique. Quand plusieurs épisodes ont été coproduits pour la même fiction, nous avons tenu compte de la date du premier épisode coproduit.

${ }^{7}$ Le chiffre indique la durée des épisodes en minutes. 


\begin{tabular}{|c|c|c|c|c|}
\hline $\mathrm{b}$ & & (minisérie) & & $08 / 06 / 1999$ \\
\hline 7 & Quai $n^{\circ} 1$ & $\begin{array}{l}90 \\
\text { (série) }\end{array}$ & Le père Fouettard & $06 / 03 / 1997$ \\
\hline 8 & Regards d'enfants & $\begin{array}{l}90 \\
\text { (collection) }\end{array}$ & $\begin{array}{l}\text { Mon père des jours } \\
\text { pairs }\end{array}$ & $21 / 09 / 1998$ \\
\hline 9 & Le devenir de l'homme & $\begin{array}{l}90 \\
\text { (collection) }\end{array}$ & $\begin{array}{l}\text { Un cœur pas comme } \\
\text { les autres }\end{array}$ & $21 / 09 / 1998$ \\
\hline 10 & Baldi & $\begin{array}{l}90 \\
\text { (série) }\end{array}$ & Baldi et Tini & $04 / 10 / 1999$ \\
\hline \multirow[t]{2}{*}{11} & \multirow{2}{*}{$\begin{array}{l}\text { Joséphine, ange } \\
\text { gardien }\end{array}$} & \multirow{2}{*}{$\begin{array}{l}90 \\
\text { (série) }\end{array}$} & Une mauvaise passe & $20 / 05 / 1999$ \\
\hline & & & La vérité en face & $08 / 12 / 2001$ \\
\hline 12 & La kiné & $\begin{array}{l}90 \\
\text { (série) }\end{array}$ & L'invitée & $10 / 10 / 2000$ \\
\hline \multirow[t]{2}{*}{13} & \multirow[t]{2}{*}{ L'instit } & \multirow{2}{*}{$\begin{array}{l}90 \\
\text { (série) }\end{array}$} & Marine et Fabien & $07 / 04 / 2000$ \\
\hline & & & Le prix du mensonge & $16 / 10 / 2001$ \\
\hline 14 & Crimes en série & $\begin{array}{l}90 \\
\text { (série) }\end{array}$ & Face à face & $13 / 11 / 2002$ \\
\hline \multirow[t]{2}{*}{15} & \multirow[t]{2}{*}{ Sauveur Giordano } & \multirow{2}{*}{$\begin{array}{l}90 \\
\text { (série) }\end{array}$} & Transports dangereux & $20 / 11 / 2003$ \\
\hline & & & Descente aux enfers & $27 / 09 / 2007$ \\
\hline 16 & Rose et Val & $\begin{array}{l}90 \\
\text { (série) }\end{array}$ & Duo d'enfer & $01 / 05 / 2005$ \\
\hline 17 & Plus belle la vie & $\begin{array}{l}4 \times 26 \\
\text { (série) }\end{array}$ & Enquêtes parallèles ${ }^{8}$ & $10 / 12 / 2010$ \\
\hline 18 & Louis la brocante & $\begin{array}{l}90 \\
\text { (série) }\end{array}$ & $\begin{array}{l}\text { Louis chez les } \\
\text { Flamands }\end{array}$ & $09 / 12 / 2011$ \\
\hline \multirow[t]{2}{*}{19} & \multirow[t]{2}{*}{$\grave{A}$ dix minutes de... } & \multirow[t]{2}{*}{$\begin{array}{l}90 \\
\text { (collection) }\end{array}$} & $\begin{array}{l}\text { À dix minutes de nulle } \\
\text { part }\end{array}$ & $17 / 04 / 2011$ \\
\hline & & & $\begin{array}{l}\text { A dix minutes des } \\
\text { naturistes }\end{array}$ & $04 / 03 / 2012$ \\
\hline
\end{tabular}

Comme nous l'avons précisé, notre intention n'était pas de confronter les fictions à une liste de stéréotypes belges. D'abord parce que cette liste serait compliquée à établir. C'est donc une démarche plus inductive qui est utilisée : nous avons repéré des éléments concernant les lieux présentés, les milieux et les personnages intervenant dans les récits, les intrigues et les thèmes abordés, les traits typiques utilisés dans les intrigues et les séquences où la Belgique ou les Belges étaient explicitement discutés. Cela ne signifie évidemment pas que nous ne connaissions pas certains stéréotypes (notamment le prétendu accent belge très utilisé par les humoristes français) et que nous n'avions pas d'intuitions préalables (par exemple que les lieux touristiques reconnaissables allaient symboliser le pays).

8 Il s'agit de quatre épisodes rassemblés sous un même titre et diffusé lors d'un primetime de la série. Régulièrement, Plus belle la vie propose des épisodes spéciaux qui sont alors isolés de la programmation habituelle en journée. Ces épisodes présentent des intrigues spéciales, notamment celle qui se déroule à Bruxelles. 
Afin de récolter les données, nous avons visionné une fois chaque épisode sélectionné. Nous avons consigné les éléments intéressants au fil de l'épisode. Nous n'avons pas procédé à une étude plan par plan des images ou à une retranscription systématique des dialogues.

L'état de l'art nous permet d'énoncer quelques hypothèses qui structureront la partie empirique de ce texte.

- Les représentations sociales sont incontournables dans la fiction et la télévision (Boyer, Veyrat-Masson), nous partons du principe qu'elles seront présentes dans le corpus.

- Jean-Pierre Esquenazi souligne que les programmes télévisuels ont une double nature : ils sont des "produits" et des "discours". Les séries sont françaises et les épisodes sont écrits par des scénaristes français dans le cadre d'une coproduction. Nous pensons que les récits présenteront des stéréotypes énoncés par des Français sur des Belges. En ce sens, les fictions dévoilent l'état de l'image contemporaine de la Belgique en France (ce qui fait débat, ce qui est à la mode...). La collaboration entre les deux chaînes pourrait cependant autant clicher les stéréotypes si les Belges se conforment aux attentes des Français (le cercle vicieux d'Isabelle VeyratMasson), que les renouveler (d'autant plus si c'est réellement l'occasion d'une coécriture).

- Les représentations sociales font consensus et deviennent des évidences (Jodelet), des mi-croyances (Flahaut). Mais comme nous sommes belges et que les stéréotypes sont énoncés par des étrangers, il nous semble que nous les repérerons. Isabelle Veyrat-Masson a remarqué que les stéréotypes énoncés par autrui étaient non seulement repérés, mais rejetés.

- Les représentations sont des schématisations (Jodelet), nous énonçons l'hypothèse que le pays et les citoyens seront réduits à quelques traits (comme les monuments de Marseille listés par Céline Bryon-Portet). Comme cette dernière, nous pensons que les caractéristiques utilisées par les séries seront les plus populaires.

- Sylvaine Marandon apporte un point pertinent quand elle constate que les stéréotypes caractérisent des personnages secondaires pittoresques. Nous vérifierons où se situent les Belges. Sont-ils des personnages principaux (potentiellement plus nuancés) ou des seconds couteaux (vraisemblablement plus clichés)?

\footnotetext{
${ }^{9}$ La plupart des séries ne sont pas disponibles en DVD. Nous avons pu visionner les épisodes à la RTBF et nous tenons à remercier le personnel du service fiction pour leur accueil.
} 
- Les séries présentées mettent en scène des héros français qui généralement voyagent dans l'Hexagone. Cet épisode coproduit est donc une exception et nous postulons que l'excursion en Belgique est un élément inhabituel. Ceci pourrait favoriser des représentations exotiques.

- Il apparaît à la lecture de l'état de l'art que plusieurs stéréotypes peuvent coexister à un moment donné soit parce que plusieurs niveaux de population ont une connaissance différente de l'objet à décrire (Crouzet), soit parce que différentes fictions proposent des imaginaires différents (Veyrat-Masson). Est-ce le cas pour la Belgique?

- Enfin, si les stéréotypes nationaux se développent dans l'interaction entre peuples, ils disent autant de choses sur les personnes croquées que sur les personnes énonçant le propos. Les séries pourraient révéler l'état des relations entre la France et la Belgique. Concernant ce dernier point, cependant, une précision nous paraît essentielle. Si Thomas Glesener et François Crouzet parviennent à dégager l'état des relations entre, respectivement, Espagnols et Flamands, et Français et Britanniques, c'est parce qu'ils adoptent une perspective historique. Il n'est pas certain que nous serons capables d'effectuer ce genre d'analyse sur l'époque contemporaine.

\section{La Belgique}

Pour cette partie concernant les lieux, nous avons repéré les lieux qui ont une empreinte belge particulière comme les monuments ou les maisons bruxelloises typiques. Nous avons également répertorié les endroits mis en scène (campagne/ville, les villes, etc.). Enfin, nous avons consigné les jugements émis à l'encontre de ces emplacements. La question sous-jacente à ce travail est : que montre-t-on de la Belgique ? Nous constatons que la Belgique est surtout urbaine et résumée par Bruxelles, que les lieux de passages et les monuments sont les plus montrés et que les personnages commentent assez peu la Belgique.

\section{a) La Belgique $=$ Bruxelles}

La capitale est, de loin, la ville qui est la plus représentée dans les épisodes étudiés. Elle est présente dans quatorze d'entre eux. Dans onze cas, Bruxelles est même le seul lieu belge montré ${ }^{10}$ ( $c f$. le tableau 2). Dans trois fictions, les personnages se déplacent en Belgique. Les intrigues

\footnotetext{
10 Même si des lieux français peuvent être montrés, notamment en début d'épisode dans
} une séquence qui justifie le voyage. 
emmènent alors les héros dans des villes wallonnes (Louvain-la-Neuve et Nivelles) ou flamandes (Anvers). Le Devenir de l'homme alterne des séquences dans la capitale et d'autres dans une petite ville de la côte belge où la grandmère réside. Seules deux fictions boudent complètement la capitale. Lonis la brocante se déroule exclusivement à Bruges. L'intrigue de Quai $n^{\circ} 1$ est située en Flandre-Occidentale.

Cela ne signifie évidemment pas que ce soit les seuls lieux présentés. En effet, plusieurs séries débutent en France dans une séquence où le héros est envoyé en Belgique (Nestor Burma, R.I.F., Crimes en série, Baldi, La Kinê). Deux séries alternent des séquences à Bruxelles et d'autres en France (A dix minutes des naturistes et Plus belle la vie).

Bruxelles jouit probablement de plusieurs avantages : il s'agit d'une ville connue internationalement; en tant que capitale elle symbolise directement le pays ; elle a l'avantage d'être à majorité francophone (ce qui est linguistiquement préférable pour les Français) ou bilingue (et donc les protagonistes peuvent être confrontés à des interlocuteurs parlant le français avec une touche exotique de flamand).

On trouve également des explications narratologiques au choix de Bruxelles. Dans Plus belle la vie, le commissaire Sara Douala est en stage à Interpol. Les autres personnages enquêtent dans le monde du lobbying européen. Ces deux éléments expliquent leur présence en Belgique. Victor Novak étant un instituteur remplaçant, il ne peut intervenir que quand des établissements dépendant des institutions françaises. Or il n'existe que deux lycées français en Belgique: un à Anvers (dans un contexte néerlandophone difficilement compréhensible pour les téléspectateurs français) et un à Bruxelles. Nestor Burma investigue dans le milieu de la haute finance actif principalement à Bruxelles en Belgique. L'héroïne de La Kiné soigne l'épouse d'un éditeur fortuné qui est implanté dans la capitale. Bruxelles est centrale, bien desservie par les transports, au cœur de la vie culturelle, on peut donc comprendre qu'il ait choisi cette situation idéale pour son entreprise.

Ces justifications narratologiques permettent également de comprendre le choix de Bruges et de la Flandre-Occidentale dans deux autres séries. Dans Louis la brocante, le protagoniste participe à un concours de peinture où les participants doivent copier le volet d'un triptyque de Hans Memling. C'est à Bruges que le peintre a terminé sa vie et au musée Saint Jean que certaines peintures sont exposées. Si les scénaristes auraient pu choisir un autre peintre, la Belgique est probablement associée aux primitifs flamands dans l'esprit des téléspectateurs. Et il n'est donc pas incongru que Louis Roman se retrouve à Bruges. Le commissaire Marie Saint-Georges s'intéresse au trafic d'hormones (Quai $n^{\circ} 1$ ). L'épisode rappelle fortement 
un fait réel : l'assassinat de Karel van Noppen, un inspecteur chargé du contrôle antihormones en Flandre ${ }^{11}$. L'épisode se déroule exactement dans cette aire géographique qui se trouve près de la frontière française et de Lille, ce qui explique que certains protagonistes s'expriment aussi bien en français qu'en flamand.

Les autres intrigues auraient par contre pu se dérouler n’importe où en Belgique. Joséphine aide une mère célibataire sans emploi qui tombe dans la prostitution (Joséphine ange gardien), le tueur en série que Thomas Berthier traque n'est pas spécifiquement relié à Bruxelles (Crimes en série), les adolescents fugueurs interceptés par un pédophile auraient pu atterrir ailleurs (R.I.F.) par exemple.

En résumé, Bruxelles est un lieu surreprésenté dans les épisodes belges des séries françaises. Cette récurrence n'est justifiable que dans certains cas seulement. Par ailleurs, les endroits que les téléspectateurs visitent sont majoritairement citadins : Bruxelles, Bruges, Roeselare, Nivelles, Anvers et Louvain-la-Neuve. C'est également le cas des lieux cités: Anvers, Linkebeek et Wavre. Seuls Le devenir de l'bomme et Quai $n^{\circ} 1$ font exception et nous emmènent dans la campagne flamande.

Tableau 2 : Les villes représentées dans les épisodes étudiés

\begin{tabular}{|c|c|c|}
\hline \multicolumn{2}{|c|}{ Uniquement Bruxelles montrée } & Autres lieux cités \\
\hline \multicolumn{2}{|c|}{ À dix minutes de... } & - \\
\hline \multicolumn{2}{|l|}{ Baldi } & - \\
\hline \multicolumn{2}{|l|}{ C'est mon histoire } & - \\
\hline \multicolumn{2}{|l|}{ Crimes en série } & - \\
\hline \multicolumn{2}{|l|}{ Joséphine, ange gardien } & - \\
\hline \multicolumn{2}{|l|}{ L'instit } & $\begin{array}{l}\text { Linkebeek et } \\
\text { Anvers }\end{array}$ \\
\hline \multicolumn{2}{|l|}{ La kiné } & - \\
\hline \multicolumn{2}{|l|}{ Le R.I.F. } & Wavre \\
\hline \multicolumn{2}{|l|}{ Nestor Burma } & - \\
\hline \multicolumn{2}{|l|}{ Plus belle la vie } & - \\
\hline \multicolumn{2}{|l|}{ Regards d'enfants } & - \\
\hline \multicolumn{2}{|c|}{ Bruxelles et d'autres lieux montrés } & Autres lieux cités \\
\hline Le devenir de l'homme & Bruxelles, Flandre & - \\
\hline Les Renseignements généraux & $\begin{array}{l}\text { Bruxelles, Louvain-la-Neuve, } \\
\text { Anvers }\end{array}$ & - \\
\hline Sauveur Giordano & Bruxelles, Nivelles & - \\
\hline \multicolumn{2}{|c|}{ Autres lieux que Bruxelles } & Autres lieux cités \\
\hline Louis la brocante & Bruges & - \\
\hline Quai n¹ & $\begin{array}{l}\text { Roeselare, Lichtervelde, } \\
\text { Flandre, Ciney (le nom de la } \\
\text { ville n’est pas cité) }\end{array}$ & - \\
\hline
\end{tabular}

11 Pour plus d'informations lire : http://www.liberation.fr/monde/1995/02/28/unveterinaire-belge-karel-van-noppen-victime-de-la-mafia-des-hormones 121890. 


\section{b) Toutes les autoroutes mènent en Belgique}

Le voyage en Belgique est parfois précédé d'une séquence où le personnage est envoyé en Belgique. Un ami appelle Nestor Burma à la rescousse. Le R.I.F. est chargé de rechercher les adolescents fugueurs et commence par les chercher en France avant d'apprendre qu'ils ont été repérés en Belgique. Anne Belfond sauve Carole d'une chute en montagne avant de la rejoindre à Bruxelles pour aider à sa rééducation ( La kiné). Thomas Berthier se réveille en famille avant de recevoir un cadeau morbide d'un tueur en séries (Crimes en série). Colette demande l'aide de Baldi pour retrouver sa petite fille qui a fugué (Baldi).

En début d'épisode (dès le générique, la première séquence ou après la scène française) le personnage se déplace vers la Belgique. Joséphine (Joséphine ange gardien) passe par un poste-frontière qui n'a d'autre fonction que de marquer le passage vers le plat pays. En effet, Joséphine ayant la faculté de se téléporter où elle le souhaite, elle aurait pu directement “atterrir" à Bruxelles. La première séquence de L'Instit présente toujours Victor Novak arrivant sur les lieux de sa nouvelle affectation en moto. Anne Belfond arrive à l'aéroport, l'équipe de R.I.F. fait plusieurs allersretours en train. C'est ce qui explique la présence massive d'autoroutes, de routes, de tunnels bruxellois, de gares, d'aéroports dans les lieux publics répertoriés ainsi que les véhicules (séquences en voiture, moto, train) et les panneaux routiers à l'image. Il faut remarquer que si les gares sont réelles (la gare du Nord est surreprésentée), les aéroports sont souvent fictifs.

Dans une analyse de trois séries françaises - Louis la brocante, Famille d'accueil et Un Village français -, Benoît Lafon s'intéresse également aux séquences d'ouverture et il constate aussi qu'elles présentent un déplacement (86). Cependant, dans son corpus, la route est rurale et ces scènes sont l'occasion de présenter la beauté de la campagne française. On peut donc établir que la scène de déplacement est une caractéristique des séries où le héros voyage (soit de manière récurrente comme Victor Novak ou ponctuelle comme Sauveur Giordano). Nos épisodes ne font pas exception. Par contre, il apparait que quand le personnage se déplace en France, c'est vers des zones rurales alors que, quand il change de pays, il visite la capitale.

\section{c) L'Atomium $=$ La Belgique}

La Belgique est présente de deux façons dans les épisodes : dans des images servant de transition entre deux séquences et au sein des scènes en tant que telles. Dans le premier cas, les lieux ne sont pas reliés à 
l'intrigue, ils symbolisent le pays ou la ville. Dans le second cas, ils servent majoritairement de décor aux dialogues et aux actions.

Le premier cas regroupe exclusivement des bâtiments emblématiques ( $c f$. tableau 3). L'Atomium et le Palais de justice sont les plus souvent utilisés pour caractériser la capitale. On remarque également la Bourse, le musée du Cinquantenaire, la cathédrale Saint Michel, le Palais royal, le Mont des arts et le Sablon. La plupart du temps, les lieux sont filmés en plan large, rapidement et constituent des séquences d'illustration. Parfois le héros est présent à l'image : Nestor Burma passe devant la Colonne du soldat inconnu, Colette emmène Tini dans le café typique À la Mort Subite (Baldi). Renseignements généraux présente un cas intéressant de séquence touristique. Roger Goupil prend le temps de flâner dans le centre historique. Il visite la rue des Bouchers, la Grand Place où il effleure la statue d'Éverard t'Serclaes mourant qui porte bonheur. Ceci est assez révélateur de la fonction de ces images de monuments célèbres : établir avec certitude que l'on se trouve en Belgique et particulièrement à Bruxelles.

D'autres endroits participent plus profondément aux actions. L'hôtel Métropole est le lieu de rendez-vous manqués entre Goupil et son fils dans Renseignements généraux, il est la base de Nestor Burma. Le quartier européen est régulièrement représenté dans Plus belle la vie et $\dot{A}$ dix minutes des naturistes qui développent des intrigues en lien avec les institutions européennes. Il est également filmé dans R.I.F., mais la série a été réalisée avant la construction du Parlement au moment où les maisons étaient petit à petit détruites. Le quartier était alors une zone défavorisée de Bruxelles où les enquêteurs recherchent les adolescents fugueurs. La protégée de Joséphine, qui se prostitue pour arrondir les fins de mois, évolue dans les quartiers chauds qui jouxtent la gare du Nord. Le quartier Matonge, rassemblant les immigrés d'origine africaine, est au centre de plusieurs séquences de Baldi. Enfin, certaines rues moins connues, mais typiques de l'architecture ou des quartiers bruxellois sont régulièrement filmées dans L'Instit (les maisons avec un entresol et les pièces en enfilade de Ixelles ou Uccle), A dix minutes des naturistes (le quartier cossu proche des institutions européennes), c'est mon bistoire (Schaerbeek), Regards d'enfants (les quartiers cossus) ou R.I.F. (le quartier du canal).

La ville de Bruges subit le même sort dans Lonis la brocante. Seul le centre historique y est montré : la place principale, les canaux, les maisons flamandes et leur façade en escalier, le béguinage. La série fait explicitement référence au tourisme puisqu'on voit les visiteurs photographier la ville, on aperçoit les bateaux qui les emmènent au fil des canaux ou les calèches qui permettent de faire le tour des rues typiques. Maryvonne, l'épouse de Louis, visite la ville par ce dernier moyen. 
Quai $n^{\circ} 1$ constitue dès lors une exception puisque cette série se déroule dans une région moins connue de Belgique et moins touristique. Les images de transition montrent les petites gares (la fiction est centrée sur la police des chemins de fer), la campagne et les corons flamands.

La Belgique est donc souvent résumée à quelques lieux touristiques. Il s'agit de monuments que les Français pourront identifier rapidement comme appartement au pays ou à Bruxelles. Ces images ont donc pour fonction de situer l'intrigue et non de proposer un endroit typiquement belge où développer une intrigue en lien avec les habitants. Seules les rues et les habitations parfois filmées permettent de dépasser l'image d'Épinal.

Céline Bryon-Portet s'intéressant à la construction de l'image de Marseille dans Plus belle la vie note également la présence de lieux symboliques: Notre-Dame de la garde, le Vieux port, la prison des Baumettes, la plage du Prado, le Parc Borély... («Les productions télévisées » \$6). Elle signale que cette présentation précise des lieux participe de l'effet de réel dont bénéficie la série ("La dimension politique » 105). Simultanément, cela participe aussi à véhiculer une image réductrice de la ville. «[...] Plus belle la vie livre une représentation de la cité phocéenne réduite à ses symboles les plus populaires » («Les productions télévisées » \$20).

$\mathrm{La}$ référence faite au tourisme au moment d'évoquer les monuments bruxellois ou les promenades de Goupil (Renseignements généraux) ou Maryvonne (Louis la brocante) trouve également un écho dans l'article de Céline Bryon-Portet. En effet, l'auteure constate que Plus belle la vie a accru le capital sympathie de Marseille ("Les productions télévisées » \$18). Les visiteurs qui passent par l'office du tourisme demandent où se trouve la place du Mistral qui est centrale dans la série, mais qui n'existe pas dans la réalité ("Les productions télévisées»\$23). Par contre, elle postule que la série a moins d'impact sur les habitants de la cité. «Cela est d'autant plus vrai qu'aujourd'hui l'attente des habitants d'un territoire se situe non pas tant au niveau symbolique qu'au niveau pratique, raison pour laquelle les médias locaux ont peu à peu délaissé l'information de nature politique [...] au profit de l'information-service (Paillart, 1993).» («Les productions télévisées » \$17).

On peut également penser que les monuments bruxellois remplissent cette double fonction contradictoire d'effet de réel et de clichage. Si cela rencontre probablement les attentes du public français, les Belges doivent y être un peu moins sensibles. L'omniprésence de l'Atomium, par exemple, semble étrange tant le monument est extérieur à 
Bruxelles et peu visité par les Belges. Par contre, pour un Français, il doit faire écho à la tour Eiffel et être donc très lisible.

Tableau 3 : Lieux représentés dans les épisodes étudiés

\begin{tabular}{|c|c|c|}
\hline Séries & Villes & Lieux publics \\
\hline $\begin{array}{l}\grave{\text { A dix minutes }} \\
\text { de... }\end{array}$ & Bruxelles & $\begin{array}{l}\text { Une rue cossue de Bruxelles, les rues du centre } \\
\text { historique bruxellois, le Berlaymont, le parlement } \\
\text { européen }\end{array}$ \\
\hline Baldi & Bruxelles & $\begin{array}{l}\text { Atomium, les toits de Bruxelles, la Grand place, } \\
\text { des boulevards bruxellois, le quartier Matonge, la } \\
\text { gare du Nord, l'hôpital Saint Pierre, le café A la } \\
\text { Mort Subite }\end{array}$ \\
\hline C'est mon histoire & Bruxelles & Rues de Schaerbeek \\
\hline Crimes en série & Bruxelles & $\begin{array}{l}\text { Le palais de justice, hôtel Métropole, une } \\
\text { brasserie }\end{array}$ \\
\hline $\begin{array}{l}\text { Joséphine, ange } \\
\text { gardien }\end{array}$ & Bruxelles & $\begin{array}{l}\text { Vue aérienne de Bruxelles avec l'Atomium, une } \\
\text { rue bruxelloise, le quartier chaud près de la gare } \\
\text { du Nord }\end{array}$ \\
\hline \multirow[t]{2}{*}{$\begin{array}{l}\text { Le devenir de } \\
\text { l'homme }\end{array}$} & Flandre & $\begin{array}{l}\text { Campagne et canaux flamands, une rue avec un } \\
\text { moulin }\end{array}$ \\
\hline & Bruxelles & Étangs d'Ixelles, CHU Bruxelles Sud \\
\hline L'instit & Bruxelles & $\begin{array}{l}\text { Autoroutes, rues de Bruxelles, tunnels, le palais } \\
\text { de justice, le Mont des arts, la cathédrale Saint } \\
\text { Michel, le Sablon, un quartier plus excentré, un } \\
\text { commerce dans une maison bruxelloise typique, } \\
\text { le lycée français, un cimetière }\end{array}$ \\
\hline La kiné & Bruxelles & $\begin{array}{l}\text { La cathédrale Saint Michel, des rues de Bruxelles, } \\
\text { le palais royal, le centre hospitalier d'Etterbeek }\end{array}$ \\
\hline \multirow{3}{*}{$\begin{array}{l}\text { Les } \\
\text { Renseignements } \\
\text { généraux }\end{array}$} & Bruxelles & $\begin{array}{l}\text { Autoroutes, Atomium, hôtel Métropole, les } \\
\text { restaurants rue des bouchers, la Grand place }\end{array}$ \\
\hline & $\begin{array}{l}\text { Louvain-la- } \\
\text { Neuve }\end{array}$ & $\begin{array}{l}\text { Routes sous la dalle, un bâtiment à appartements, } \\
\text { halles universitaires (fresque), rue des Wallons }\end{array}$ \\
\hline & Anvers & Quartier du port \\
\hline Le R.I.F. & Bruxelles & $\begin{array}{l}\text { La gare de Schaerbeek, palais de Justice et place } \\
\text { Poelaert, la gare du Nord, la bourse, les quartiers } \\
\text { européens (maisons expropriées pour construire } \\
\text { le Parlement), le canal, le boulevard Jacqmain, le } \\
\text { quartier chaud près de la gare du Nord, le musée } \\
\text { du Cinquantenaire }\end{array}$ \\
\hline Louis la brocante & Bruges & $\begin{array}{l}\text { Canaux, béguinage, une maison typique } \\
\text { brugeoise }\end{array}$ \\
\hline Nestor Burma & Bruxelles & $\begin{array}{l}\text { Autoroute, tunnels, gare du Nord, place de } \\
\text { Brouckère, Hôtel Métropole, colonne du soldat } \\
\text { inconnu }\end{array}$ \\
\hline Plus belle la vie & Bruxelles & $\begin{array}{l}\text { Le Mont des arts, le parlement européen, le } \\
\text { palais de justice, Atomium, une rue bruxelloise }\end{array}$ \\
\hline \multirow[t]{4}{*}{ Quai n¹ } & Roeselare & Gare \\
\hline & Lichtervelde & Gare \\
\hline & Flandre & $\begin{array}{l}\text { Corons flamands, la campagne flamande, } \\
\text { Schuiferskapelle, des fermes }\end{array}$ \\
\hline & $\begin{array}{l}\text { Ciney (le nom } \\
\text { de la ville n'est }\end{array}$ & Le marché aux bestiaux \\
\hline
\end{tabular}




\begin{tabular}{|l|l|l|}
\hline & pas cité) & \\
\hline Regards d'enfants & Bruxelles & $\begin{array}{l}\text { Quartier cossu, grands boulevards arborés, } \\
\text { quartier plus périphérique et populaire }\end{array}$ \\
\hline Sauveur Giordano & Bruxelles & $\begin{array}{l}\text { Clinique Saint-Pierre, une ue de Bruxelles, le } \\
\text { palais de justice, l'Onem }\end{array}$ \\
\cline { 2 - 3 } & Nivelles & Routes entre Nivelles et Bruxelles \\
\hline
\end{tabular}

\section{d) Les lieux, ce qu'on en dit}

Il y a étonnement assez peu de discours sur les lieux visités. Bruxelles est l'objet de quelques commentaires plutôt superficiels. Joséphine trouve que c'est une grande ville «mais c'est joli ». Alors qu'il espérait devoir partir pour la Guyane afin de retrouver les deux adolescents fugueurs, un policier de R.I.F. déclare que Bruxelles, c'est moins exotique. De retour à Paris, les inspecteurs émettent un avis partagé sur la capitale belge : «C'est une drôle de ville. La Grand place, c'est comme une scène de théâtre. »Ce propos ne sera pas explicité. Tous les personnages sans exception, même quand ils vivent en Belgique depuis longtemps comme les concierges d' $A$ dix minutes des naturistes, prononcent "Bruxelles" à la française, c'est-à-dire avec un " $\mathrm{x}$ " (BruXelles) et non un "s" (BruSSelles).

Les commentaires sur le pays portent sur la nourriture ou sont ironiques. Dans Baldi, Colette commande une pils, car «après tout, on est dans le pays de la bière ». Hélène, la secrétaire de Nestor Burma, s'extasie sur le petit déjeuner servi à l'hôtel : "C'est merveilleux toutes ces petites choses ». Son patron lui rétorque : "C’est la Belgique Hélène ». On ne saura pas vraiment ce que cela signifie. D'autres discours sont plus désabusés. Dépité par l'état de l'appartement qu'ils louent à un policier belge, un collègue de Thomas Berthier (Crimes en série) s'exclame: «Tu parles d'un pays! C'est comme le Manneken-Pis, tellement petit que je suis passé quatre fois devant ». Il est vrai que la statue n'est pas volumineuse et que les touristes la cherchent souvent un moment avant de la trouver.

Les autres endroits visités par les séries françaises ne récoltent pas beaucoup plus de discours. Maryvonne (Louis la brocante) trouve que «la Venise du Nord» est «romantique» et que le béguinage est joli. En déplacements dans les villages industrieux de Flandre, Marie Saint-Georges (Quai $\left.n^{\circ} 1\right)$ déclare que les villes de frontière sont «baroques ». Plus tard, alors que les protagonistes visitent le marché aux bestiaux de Ciney (dont le nom n'est jamais cité), le vétérinaire souligne que c'est l'un des plus grands d'Europe.

Bref, les commentaires sur les lieux portent sur des clichés : la Grand place, Manneken-Pis, la bière ou la romantique Venise du Nord. Les autres propos sont superficiels (c'est joli) ou mystérieux (la Grand place est une 
scène de théâtre, les villes frontière sont baroques). La raison du manque de discours sur le pays est peut-être explicitée par l'un des personnages de R.I.F. quand il souligne que la Belgique est moins exotique que la Guyane. Les lieux sont proches de ce que les protagonistes connaissent en France et donc ils génèrent peu de commentaires. Pourtant, dans cette même série, un personnage souligne malgré tout un décalage ("C'est une drôle de ville »). Ceci explique peut-être la présence des poncifs touristiques habituels. L'on utilise les stéréotypes pour symboliser l'ailleurs ('étranger et l'autre) tout en proposant un paysage connu (cet ailleurs correspond à l'image traditionnelle des Belges).

\section{Les Belges}

\section{a) Héros et personnages importants français}

La majorité des épisodes qui se déroulent en Belgique présentent un héros français (cf. tableau 4). C’est évidemment logique, car ces séries ont déjà un héros bien identifié qui est le moteur du récit. Stéphane Benassi a répertorié les éléments variants et invariants dans les fictions plurielles afin de distinguer les feuilletons des séries. Selon lui, la récurrence du héros est l'une des règles incontournables de la logique sérielle. Avec le passage de la frontière, certaines choses peuvent exceptionnellement changer (les lieux par exemple), mais pas le personnage principal.

Cette règle n'existant plus pour les collections, c'est dans ce genre que les personnages belges se retrouvent. Dans C'est mon histoire et Regards d'enfants, tous les personnages sont Bruxellois sans aucune exception. Dans Le devenir de l'homme, la nationalité des protagonistes n'est pas précisée.

Tableau 4 : Les personnages principaux dans les épisodes étudiés

\begin{tabular}{|l|l|}
\hline \multicolumn{2}{|c|}{ Personnage principal français } \\
\hline Nestor Burma & Nestor Burma \\
\hline $\begin{array}{l}\text { Les Renseignements } \\
\text { généraux }\end{array}$ & Roger Goupil \\
\hline Le R.I.F. & Commissaire Keller, Isa \\
\hline Quai n¹ & Marie Saint-Georges \\
\hline Baldi & Baldi \\
\hline Joséphine, ange gardien & Joséphine \\
\hline La kiné & Anne Belfond \\
\hline L'instit & Victor Novak \\
\hline Crimes en série & Thomas Berthier \\
\hline Sauveur Giordano & Sauveur Giordano \\
\hline Plus belle la vie & $\begin{array}{l}\text { Sybille Cassagne, Ninon Chaumette, Sara Douala, Benoit } \\
\text { Cassagne, Estelle Cantorelle, Djawad Sangha }\end{array}$ \\
\hline Louis la brocante & Louis Roman \\
\hline A dix minutes de... & Valérie et Michel, Laure et Patrick \\
\hline & Personnage principal belge \\
\hline
\end{tabular}




\begin{tabular}{|l|l|}
\hline C'est mon histoire & Aline \\
\hline Regards d'enfants & Laura, Madeleine, Cécile, Patrick et Denise \\
\hline \multicolumn{2}{|c|}{ Nationalité non précisée } \\
\hline Le devenir de l'homme & La famille \\
\hline
\end{tabular}

Si la figure du héros est évidemment intouchable dans les séries, les personnages secondaires représentent par contre un groupe plus facilement modifiable. On pouvait donc postuler, puisqu'il s'agit d'une coproduction avec la RTBF, que les Belges allaient endosser des rôles, probablement non récurrents, mais malgré tout importants au sein de l'épisode. Ces protagonistes pouvaient par exemple être des interlocuteurs exceptionnels pour le héros. Ce n'est pas le cas. Dans les treize séries analysées ${ }^{12}$, seulement quatre, peut-être cinq si on tient compte de Colette, exploitent cette possibilité (cf. tableau 5).

Dans trois séries, le héros est secondé par un enquêteur belge, qu'il soit professionnel (l'inspecteur Wauthose dans R.I.F., Michel Fournier dans Quai $n^{\circ} 1$ ) ou pas ('amie de Julie dans Sauveur Giordano). Le personnage belge remplace alors certains personnages d'assistants habituels qui, contrairement au héros, n'ont pas fait le voyage. En effet, dans Quai $n^{\circ} 1$, le collègue habituel de Marie Saint George a un rôle plus ténu lors de cet épisode. Dans R.I.F., une partie de l'équipe reste en France et l'inspectrice belge seconde les deux policiers français qui se sont déplacés.

Dans La Kiné, Anne Belfond n'est pas véritablement secondée par un personnage belge, car il ne s'agit pas d'une enquête. Il s'agit plutôt d'une histoire romantique compliquée qui se termine en impasse. Mais l'intrigue suggère néanmoins que Mathieu Morel est un homme désormais important dans la vie de l'héroïne. Le personnage secondaire est donc essentiel.

Le cas de Colette (Baldi) est plus ambigu. Colette est Belge, mais personne ne semble le savoir avant cette aventure bruxelloise. Elle n'est pas vraiment un personnage spécifiquement créé pour l'épisode belge, car elle est déjà apparue dans une intrigue précédemment. Pourtant, elle est jouée par Annie Cordy, une actrice identifiée comme typiquement Belge par les Belges et les Français. Cette figure assure également le rôle de guide en emmenant les protagonistes dans les cafés et quartiers de la capitale et de traducteur des mœurs belges. Colette apparaît donc à la fois comme un personnage habituel de la série et une Belge typique.

Dans toutes les autres fictions, c'est-à-dire huit sur les treize étudiées, soit le héros n'est pas accompagné par un Belge (Nestor Burma,

12 Nous laissons de côté Le devenir de l'homme, C'est mon histoire et Regards d'enfants qui présentent soit des personnages sans nationalité ou des personnages uniquement belges. 
Joséphine ange gardien, L'instit, Plus belle la vie), soit le personnage secondaire reste un Français parfois de manière un peu artificielle. Dans Crimes en séries, par exemple, Thomas Berthier enquête avec des policiers belges dirigés par une commissaire talentueuse, mais il s'agit d'une Française expatriée. Gabrielle Jabon est d'ailleurs une ancienne petite amie de Thomas. Le principe de la collection, $A 10$ minutes de... est de montrer deux familles qui échangent leurs maisons et de voir les personnages évoluer dans un environnement qu'ils ne connaissent pas. Ce principe aurait permis de montrer des Belges et des Français assez facilement. Pourtant le couple habitant en Belgique est composé de deux expatriés français (qui travaillent comme concierges pour des députés européens français) qui échangent la maison de leurs patrons avec d'autres Français. Dans Nestor Burma ou Renseignements généraux, les personnages secondaires habituels (la secrétaire, le commissaire Faroux, le fils de Goupil) se déplacent également à Bruxelles pour des raisons plus ou moins convaincantes.

Tableau 5 : Les personnes secondaires représentées dans les épisodes étudiés

\begin{tabular}{|c|c|c|}
\hline \multirow[t]{2}{*}{ Séries } & \multicolumn{2}{|c|}{ Principaux interlocuteurs } \\
\hline & Belges & Français (sauf exception) \\
\hline$\grave{A}$ dix minutes de... & $\begin{array}{l}\text { Les voisins, les autorités de la } \\
\text { ville de Bruxelles }\end{array}$ & $\begin{array}{l}\text { Les eurodéputés propriétaires de } \\
\text { la maison, les personnes } \\
\text { rencontrées à l'île du couchant }\end{array}$ \\
\hline Baldi & $\begin{array}{l}\text { Colette, Stéphanie dite Tini } \\
\text { (petite fille de Colette), Solange } \\
\text { (belle-fille de Colette), Stan } \\
\text { (petit ami) }\end{array}$ & $\begin{array}{l}\text { Zaiko (adolescent qui } \\
\text { accompagne Colette) }\end{array}$ \\
\hline C'est mon histoire & $\begin{array}{l}\text { Le père de l'héroïne, son amie, } \\
\text { son petit ami }\end{array}$ & \\
\hline Crimes en série & $\begin{array}{l}\text { Eric Debaeker (le tueur), } \\
\text { Corinne Debaecker (l'épouse } \\
\text { du tueur), Luc Meulemans } \\
\text { (policier belge), Jacques } \\
\text { Mercier (présentateur de Fort en } \\
\text { têtes) }\end{array}$ & $\begin{array}{l}\text { Gabrielle Jabon (commissaire en } \\
\text { Belgique, mais d'origine } \\
\text { française), Maud Berthier } \\
\text { (épouse de Thomas), Pierre } \\
\text { Denard, Primprennelle et } \\
\text { Fankenstein (équipe de Berthier) }\end{array}$ \\
\hline $\begin{array}{l}\text { Joséphine, ange } \\
\text { gardien }\end{array}$ & $\begin{array}{l}\text { Véronique Maillard (la } \\
\text { protégée de Joséphine), Chloé } \\
\text { et Vanessa Maillard (les filles } \\
\text { de Véronique), Corinne (la } \\
\text { collègue), Wadeck Maréchal (le } \\
\text { journaliste) }\end{array}$ & \\
\hline $\begin{array}{l}\text { Le devenir de } \\
\text { l'homme }\end{array}$ & $\begin{array}{l}\text { Les parents de l'héroïne, les } \\
\text { médecins }\end{array}$ & \\
\hline L'instit & & $\begin{array}{l}\text { Morgane (adolescente aidée par } \\
\text { Novak), Agnès (mère de } \\
\text { Morgane), Catherine (grand- } \\
\text { mère canadienne), Nathalie } \\
\text { (amie de Morgane, nationalité } \\
\text { non précisée), Fred (sœur de } \\
\text { Nathalie et amie de Morgane, } \\
\text { nationalité non précisée) }\end{array}$ \\
\hline
\end{tabular}




\begin{tabular}{|c|c|c|}
\hline La kiné & $\begin{array}{l}\text { Mathieu Morel (le mari de } \\
\text { Carole), Carole Morel (la } \\
\text { blessée), Madeleine } \\
\text { (domestique des Morel) }\end{array}$ & \\
\hline $\begin{array}{l}\text { Les } \\
\text { Renseignements } \\
\text { généraux }\end{array}$ & $\begin{array}{l}\text { Le commissaire Van den } \\
\text { Bergh, Vercheval (un } \\
\text { gangster), Françoise Renoir (la } \\
\text { petite-amie Laurent) }\end{array}$ & $\begin{array}{l}\text { Laurent Guimard (ancien } \\
\text { policier français résidant en } \\
\text { Belgique), Simon (fils), } \\
\text { Stéphanie (nounou de Simon) }\end{array}$ \\
\hline Le R.I.F. & $\begin{array}{l}\text { L'inspectrice Wauthose, Alain } \\
\text { Van de Kerkoove (le } \\
\text { proxénète) }\end{array}$ & $\begin{array}{l}\text { Claire et Julien (adolescents), } \\
\text { Pierre et Valérie Charlier } \\
\text { (parents), leur oncle (Français } \\
\text { résidant en Belgique), Roberto } \\
\text { Toli (malfrat, Corse), Boccello } \\
\text { (malfrat, Corse) }\end{array}$ \\
\hline Louis la brocante & $\begin{array}{l}\text { Jeff van Pamel, Monsieur van } \\
\text { Pamel (père de Jeff), Merelle } \\
\text { (aide de Jeff), Wim de Greef, } \\
\text { Maria (propriétaire de la } \\
\text { pension de famille), Monsieur } \\
\text { Til (steward de la pension de } \\
\text { famille), les dames du cours de } \\
\text { dentelle }\end{array}$ & Maryvonne \\
\hline Nestor Burma & $\begin{array}{l}\text { Monsieur Derogier (le } \\
\text { banquier), Janine Derogier (sa } \\
\text { fille), Paul Grillat (petit ami de } \\
\text { Janine), Albert Buard (le } \\
\text { collaborateur du banquier) }\end{array}$ & Hélène (secrétaire) \\
\hline Plus belle la vie & $\begin{array}{l}\text { Marc Dana (Interpol), la } \\
\text { cousine de Djawat, Xavier } \\
\text { Méresse (policier ripou) }\end{array}$ & $\begin{array}{l}\text { Laetitia Dewaere (épouse d'un } \\
\text { député européen écologiste,), } \\
\text { Charles McGregor (identité non } \\
\text { précisée), Emma McGregor } \\
\text { (identité non précisée) }\end{array}$ \\
\hline Quai nº1 & $\begin{array}{l}\text { Michel Fournier (le vétérinaire } \\
\text { qui assiste l'hérö̈ne), la femme } \\
\text { et le fils du vétérinaire, Luc } \\
\text { Fournier (le frère du } \\
\text { vétérinaire), Zwarte Piet (un } \\
\text { agriculteur), Katrien Stallaert } \\
\text { (la directrice haras), madame } \\
\text { Walravens (supérieur de } \\
\text { Michel), le commandant de } \\
\text { Wael, Marc (architecte) }\end{array}$ & $\begin{array}{l}\text { Capitaine Max Urtéguy (collègue } \\
\text { de Marie) }\end{array}$ \\
\hline Regards d'enfants & $\begin{array}{l}\text { Le professeur, la patronne du } \\
\text { café, Yvanna et son frère }\end{array}$ & \\
\hline Sauveur Giordano & $\begin{array}{l}\text { Marie (l'amie de Julie), } \\
\text { Commissaire Roosen, } \\
\text { Chauffeur du car, Clara } \\
\text { Vermeyren (victime de } \\
\text { l'accident), Fabienne Vande } \\
\text { Merssche (présentatrice du JT) }\end{array}$ & Julie Giordano \\
\hline
\end{tabular}

Si les personnages déterminants de l'histoire restent donc Français, cela ne signifie pas qu'aucun Belge n'est présent dans les fictions ( $c f$. tableau 
6). Seules deux fictions semblent présenter des personnages uniquement français (L'instit et $A$ dix minutes des naturistes). À l'autre extrême, deux séries présentent uniquement des Belges (C'est mon bistoire et Regards d'enfants). En fait, la plupart des séries en profitent pour plonger le héros ou le héros et son acolyte ou un groupe de héros dans la population belge. La Belgique représente donc la couleur locale de la semaine.

Tableau 6 : Le mélange des Belges et Français dans les épisodes étudiés ${ }^{13}$

\begin{tabular}{|c|c|c|c|}
\hline $\begin{array}{c}\text { Personnel } \\
\text { exclusivement } \\
\text { français }\end{array}$ & $\begin{array}{c}\text { Héros et } \\
\text { acolyte français } \\
+ \text { milieu belge }\end{array}$ & $\begin{array}{c}\text { Héros français }+ \\
\text { milieu belge }\end{array}$ & $\begin{array}{c}\text { Personnel } \\
\text { exclusivement belge }\end{array}$ \\
\hline $\begin{array}{l}\text { - L'instit } \\
\text { - À dix minutes des } \\
\text { naturistes }\end{array}$ & $\begin{array}{l}\text { - Nestor Burma } \\
\text { - Quai n } 1 \\
\text { - Baldi } \\
\text { - Sauveur } \\
\text { Giordano } \\
\text { - Louis la } \\
\text { Brocante } \\
\text { - } \\
\text { Renseignements } \\
\text { généraux } \\
\text { - R.I.F. } \\
\text { - Crimes en } \\
\text { séries } \\
\text { - Plus belle la vie }\end{array}$ & $\begin{array}{l}\text { - Joséphine ange } \\
\text { gardien } \\
\text { - La kiné }\end{array}$ & $\begin{array}{l}\text { - C'est mon histoire } \\
\text { - Regards d'enfants }\end{array}$ \\
\hline
\end{tabular}

\section{b) Les milieux visités}

La répartition des fictions en fonction du milieu dominant dans lequel elle se déroule correspond au genre majoritairement policier où les héros côtoient des criminels et des institutions (à commencer par les institutions policières). Dans le tableau 7, nous n'avons classé dans la catégorie "crime" que les milieux qui relèvent spécifiquement de l'illégalité. Dans les séries analysées, le crime se loge également dans d'autres milieux. Par exemple, Nestor Burma rencontre des banquiers criminels qui détournent de l'argent et assassinent pour se protéger. Mais le milieu de la finance n'est pas criminel en soi alors que, par nature, le grand banditisme l'est.

Pour la plupart des séries, on ne peut pas affirmer qu'elles se déroulent dans des milieux typiquement belges. On croise des agriculteurs, des étudiants, le personnel de musées, les travailleurs d'une société d'autocar, des employés des milieux financiers, des médecins, des prostituées, des bandits dans tous les pays.

13 Est exclue Le devenir de l'homme qui reste neutre à propos des nationalités des protagonistes. 
Deux éléments semblent pourtant intéressants. D'abord, deux séries exploitent Bruxelles en tant que capitale européenne. Les intrigues développées dans Plus Belle la vie et $A$ dix minutes des naturistes n'auraient probablement pas pu se dérouler ailleurs, car c'est à Bruxelles que se concentre les lobbies et les députés européens.

Le deuxième cas est plus ambigu. R.I.F. suit une enquête pour retrouver deux fugueurs qui tombent dans un réseau de pédophilie. Il serait évidemment erroné d'affirmer que seule la Belgique possède des pédophiles. Il faut également se garder de penser que la série surfe sur la vague de l'affaire Dutroux. En effet, si les enlèvements de certaines victimes ont eu lieu en 1995, le nom de Marc Dutroux n'est pas connu avant août $1996^{14}$. La série donc n'a pas pu s'inspirer de ce dossier judiciaire, car l'épisode est diffusé en février 1996 et donc écrit bien avant. Il est difficile de trouver des informations concernant la série, car elle n'a connu que quatre épisodes. Il est donc difficile de savoir si elle présentait toujours des affaires de pédophile (il semble que non) et comment elles étaient traitées. Une hypothèse est qu'il était peut-être plus facile, pour une chaîne française, de situer ce type d'affaires en dehors du sol national. D'autres pays que la Belgique auraient alors pu faire l'affaire. Dans tous les cas, avec le recul du temps, cet épisode a une résonnance toute particulière.

Tableau 7 : Les milieux présentés dans les épisodes étudiés

\begin{tabular}{|c|c|c|c|c|}
\hline Crime & Institutions & Art/tourisme & Famille & Autres \\
\hline $\begin{array}{l}\text { Renseignements } \\
\text { généraux (grand } \\
\text { banditisme) } \\
\text { R.I.F. } \\
\text { (pédophilie) } \\
\text { Joséphine ange } \\
\text { gardien } \\
\text { (prostitution) }\end{array}$ & $\begin{array}{l}\text { Nestor Burma } \\
\text { (finance) } \\
\text { Plus belle la vie } \\
\text { (Europe) } \\
\text { À dix minutes } \\
\text { des naturistes } \\
\text { (Europe) } \\
\text { Le devenir de } \\
\text { l'homme } \\
\text { (recherche } \\
\text { médicale) }\end{array}$ & $\begin{array}{l}\text { Sauveur } \\
\text { Giordano } \\
\text { (société de, car) } \\
\text { Louis la } \\
\text { Brocante } \\
\text { (musée d'art) }\end{array}$ & $\begin{array}{l}\text { L'instit } \\
\text { La kiné } \\
\text { Crimes en } \\
\text { série }\end{array}$ & $\begin{array}{l}\text { Quai n }{ }^{\circ} 1 \\
\text { (agriculture) } \\
\text { Baldi } \\
\text { (étudiant) }\end{array}$ \\
\hline
\end{tabular}

\section{c) Héros français vs. antihéros belges?}

Les héros restent français ainsi que la plupart de leurs bras droits quand ils en ont. Cela signifie-t-il que la seule place réservée aux Belges est celle du vilain ? Onze séries étudiées présentent des enquêtes, menées par des professionnels ou non. Et donc, il y a effectivement un "malfrat" dans ces épisodes. Ces intrigues se déroulant en Belgique, souvent ce personnage est un Belge.

14 http://tempsreel.nouvelobs.com/monde/20040617.OBS1268/chronologie-de-laffaire.html. 
Pourtant, on ne peut pas tracer un parallèle évident entre Français et "bons", Belges et "mauvais". Dans Nestor Burma, par exemple, le banquier corrompu et d'extrême droite est belge, mais les victimes et les adjuvants de Nestor aussi. Dans Joséphine ange gardien, Véronique tombe petit à petit dans la prostitution, poussée par les conseils mal avisés d'une collègue qui est belge. Cependant, la victime est également belge, ainsi que les personnages qui tentent de l'aider (notamment le journaliste). Enfin, dans Quai $n^{\circ} 1$, les trafiquants d'hormones et les assassins sont belges, tout comme les adjuvants de Marie. À l'inverse, dans L'instit, tous les personnages, bons ou méchants, sont français.

Au sein de ces séries d'enquêtes, deux présentent malgré tout des discours plus manichéens. Dans R.I.F., les enquêteurs qui comptent sont français, même s'ils sont aidés par des Belges. Les victimes sont deux adolescents français en fugue et qui sont recherchés par l'équipe. Ils tombent dans un réseau de pédophilie en Belgique. Et les coupables sont soit belges, soit corses (les dialogues insistent sur ce régionalisme). Dans le prime time de Plus belle la vie qui se déroule à Bruxelles, tous les héros sont des personnages récurrents de la série. Les personnages rencontrés sont surtout des expatriés évoluant en orbite des institutions européennes. Les quelques Belges rencontrés appartiennent aux forces de l'ordre qui sont soit corrompues, soit utilisent des pratiques contestables (comme utiliser Sibylle, une adolescente, comme appât). Le temps n'est pas un facteur explicateur (les séries plus actuelles seraient plus nuancées), car R.I.F. date de 1995 et Plus Belle la vie de 2010.

Pour les quatre séries non policières ${ }^{15}$, à part $A$ dix minutes des naturistes qui présente un environnement franco-français où les Belges font de la figuration, le paysage est généralement nuancé. Anne Belfond ( $\mathrm{La}$ Kyné) évolue dans un milieu familial où elle rencontre à la fois des personnages hostiles et des personnages amicaux, dont l'amour de sa vie. C'est mon histoire présente le parcours d'une adolescente abusée par son père qui croise des opposants ou des lâches autant que des gens tentant de l'aider. Il n'y a pas vraiment de bons et de méchants dans Regards d'enfants.

\section{d) Les Belges, ce qu'on en dit}

Les discours sur les Belges sont encore plus rares que les commentaires sur les lieux. Les séries qualifient rarement la nation dans son ensemble ou même des communautés. Trois cas, assez ténus, peuvent être repérés.

15 Nous devons exclure Le devenir de l'homme dont les nationalités ne sont pas précisées. 
Un dialogue de Renseignements généraux utilise la célèbre expression de Jules César, "De tous les peuples de la Gaule, les Belges sont les plus braves ». Il s'agit d'un Belge qui explique à son homologue français la corruption des politiciens. Il s'agit donc d'une récupération ironique de la citation. Dans le même genre, un policier flamand interrogeant Louis Roman (Louis la brocante) lui fait remarquer que les Belges ne sont pas « des sauvages ", car ils appliquent la présomption d'innocence. Cette clarification suppose implicitement que l'agent a pressenti que Louis Roman (et peut-être ses compatriotes par extension) considère les Belges comme moins civilisés. Dans Baldi, quand Zaiko apprend que Colette est belge, il s'exclame : «Ca ne se voit pas ». Elle lui rétorque qu'il est français et que ça ne se voit pas non plus. L'étonnement de l'adolescent permet également de supposer qu'il devrait exister une différence physique entre les Belges et les Français.

Ces différents commentaires sont plutôt joués sur le mode comique et doivent donc être analysés prudemment. Il semble cependant exister une volonté de rappeler, via des personnages ou des remarques humoristiques, que les Belges sont proches des Français.

\section{Les particularismes nationaux}

Au fil des scénarios, des dialogues, des images, des détails rappellent que les personnages sont en Belgique. Ces éléments font, pour la plupart, partie du décor. L'utilisation des langues nous parait l'aspect le plus intéressant. Mais avant de nous lancer dans cette étude, nous allons rapidement passer en revue les autres traits typiquement belges relevés dans les épisodes. La presse, les conversions de tout genre (de la monnaie, des chiffres, du système policier), le passé colonial, la culture et la gastronomie sont beaucoup employés.

\section{a) La Belgique $=$ la bière}

Les éléments médiatiques sont parmi les plus fréquents dans les séries analysées. Leur apparition remplit des fonctions variées, depuis la simple figuration jusqu'à un rôle central dans l'intrigue. C'est le cas de l'émission Forts en têtes diffusée sur la RTBF de 1997 à 2005 et qui est l'endroit où le tueur de Crimes en séries recrute ses victimes. Dans la majorité des cas cependant, les journaux et les JT sont des moyens pratiques de diffuser de l'information sur l'enquête aux personnages et aux téléspectateurs. La plupart du temps, il s'agit de titres francophones, mais on note une apparition du Het Laatste Nieuns dans Quai $n^{\circ} 1$ qui se déroule sur le sol flamand. Les personnalités médiatiques figurent également dans les 
épisodes, soit des journalistes réels (Fabienne Vande Merssche ou Paul Germain), soit des animateurs de la chaîne (qui jouent les figurants lors d'une fête dans Plus belle la vie).

Assez traditionnellement, la gastronomie belge est également régulièrement évoquée dans les récits. La bière est de loin la plus employée (parfois appelée par le belgicisme "petite mousse»), devant les «moules frites ». Certains produits moins connus apparaissent néanmoins comme les tartines [tranches de pain] ou le sirop de Liège.

Les éléments culturels restent assez anecdotiques. Ils permettent parfois de plonger le téléspectateur dans le milieu exploré par le héros. Les aventures des personnages de Baldi les emmènent à la Saint $\mathrm{V}$, une fête étudiante, alors qu'ils s'intéressent à des jeunes. L'épisode de Louis la brocante montre régulièrement des peintures des maitres flamands ou évoque la dentelle de Bruges. L'enquête de Marie Saint George (Quai $n^{\circ} 1$ ) se déroule sur fond de la fête de Saint Nicolas ${ }^{16}$, le méchant étant d'ailleurs prénommé Zwarte Piet, jouant sur la double signification de l'expression. En effet, Zwarte Piet est à la fois le père Fouettard (l'acolyte de Saint Nicolas), mais aussi le Valet puant dans le jeu de cartes Le valet noir ${ }^{17}$. Dans d'autres cas, ces détails caractérisent un personnage. Quand on précise qu'une danseuse a travaillé avec Maurice Béjart, cela indique qu'elle était de haut niveau (Baldi). Dans Renseignements généraux, un policier possède "évidemment" un portrait de Simenon dans son bureau. D'autres allusions parsèment les dialogues et font référence à des choses connues : l'expression « le plat pays » est devenue synonyme de la Belgique depuis la chanson de Jacques Brel, les histoires belges, l'hymne national (La Brabançonne) ou nos souverains...

Dans les séries plus anciennes, les personnages passent leur temps à convertir les prix indiqués en Franc belge en leur équivalent en français. Évidemment, cette source de quiproquos a disparu avec l'entrée en vigueur de l'Euro. Régulièrement les personnages s'étonnent de l'utilisation du nonante pour désigner le chiffre 90 .

Tous ces particularismes ont clairement pour fonction d'ajouter de la couleur locale à l'épisode. Ils sont rarement significatifs dans l'intrigue. La plupart sont d'ailleurs assez clichés. L'utilisation des particularismes langagiers, tout aussi attendus, notamment le prétendu accent belge, offre par contre des scènes plus intéressantes.

Tableau 8 : L'utilisation d'éléments belges

\footnotetext{
16 L'équivalent en Belgique du Père Noël.

17 Ce jeu s'appelle aussi Le pouilleux ou Vieux garçon en France, Le puant ou Le valet noir en Belgique, La pisseuse au Québec.
} 


\begin{tabular}{|c|c|c|c|c|c|}
\hline & $\begin{array}{l}\text { Presse } \\
\text { Personnalit } \\
\text { és }\end{array}$ & Conversion & $\begin{array}{l}\text { Passé } \\
\text { colonia } \\
1\end{array}$ & Culture & $\begin{array}{l}\text { Gastrono } \\
\text { mie }\end{array}$ \\
\hline $\begin{array}{l}\text { À dix } \\
\text { minutes... }\end{array}$ & & & & & $\begin{array}{l}\text { Moules- } \\
\text { frites }\end{array}$ \\
\hline Baldi & & & & $\begin{array}{l}\text { Saint V } \\
\text { Béjart }\end{array}$ & $\begin{array}{l}\text { Pils } \\
\text { «Petite } \\
\text { mousse » } \\
\text { Tartines }\end{array}$ \\
\hline $\begin{array}{l}\text { C'est mon } \\
\text { histoire }\end{array}$ & $\begin{array}{l}\text { Jacques } \\
\text { Mercier }\end{array}$ & & & & \\
\hline $\begin{array}{l}\text { Crimes en } \\
\text { série }\end{array}$ & $\begin{array}{l}\text { Forts en } \\
\text { têtes }\end{array}$ & $\begin{array}{l}\text { Réforme des } \\
\text { polices et } \\
\text { système } \\
\text { judiciaire belge }\end{array}$ & & & \\
\hline $\begin{array}{l}\text { Joséphine } \\
\text { ange gardien }\end{array}$ & & $\begin{array}{l}\text { Conversion au } \\
\text { franc }\end{array}$ & $\begin{array}{l}\text { Référen } \\
\text { ce au } \\
\text { Congo }\end{array}$ & & \\
\hline La kiné & - & - & - & - & - \\
\hline $\begin{array}{l}\text { Le devenir de } \\
\text { l'homme }\end{array}$ & $\begin{array}{l}\text { Le Soir } \\
\text { Paul } \\
\text { Germain }\end{array}$ & $\begin{array}{l}\text { Nonante/quat } \\
\text { re-vingt-dix }\end{array}$ & & & $\begin{array}{l}\text { Sirop de } \\
\text { Liège } \\
\text { Carbonades } \\
\text { flamandes } \\
\text { Gueuze }\end{array}$ \\
\hline L'Instit & & $\begin{array}{l}\text { Conversion au } \\
\text { franc }\end{array}$ & & & \\
\hline $\begin{array}{l}\text { Louis la } \\
\text { brocante }\end{array}$ & & & & $\begin{array}{l}\text { Dentelle } \\
\text { de Bruges } \\
\text { Peinture } \\
\text { flamande } \\
\text { Famille } \\
\text { royale } \\
\text { Brabançon } \\
\text { ne }\end{array}$ & \\
\hline Nestor Burma & Le Soir & & & & $\begin{array}{l}\text { Mort Subite } \\
\text { Moules- } \\
\text { frites } \\
\text { Tarte au riz }\end{array}$ \\
\hline $\begin{array}{l}\text { Plus belle la } \\
\text { vie }\end{array}$ & $\begin{array}{l}\text { Le Soir } \\
\text { Animateurs } \\
\text { RTBF }\end{array}$ & & & & \\
\hline Quai n ${ }^{\circ} 1$ & $\begin{array}{l}\text { Le Soir } \\
\text { Het Laatste } \\
\text { Nieuws }\end{array}$ & $\begin{array}{l}\text { Conversion au } \\
\text { franc }\end{array}$ & $\begin{array}{l}\text { Référen } \\
\text { ce au } \\
\text { Rwanda }\end{array}$ & $\begin{array}{l}\text { Zwarte } \\
\text { Piet } \\
\text { Saint- } \\
\text { Nicolas } \\
\text { Plat Pays }\end{array}$ & $\begin{array}{l}\text { Mort Subite } \\
\text { Chocolats }\end{array}$ \\
\hline $\begin{array}{l}\text { Regard } \\
\text { d'enfants }\end{array}$ & - & - & - & - & - \\
\hline $\begin{array}{l}\text { Renseigneme } \\
\text { nts généraux }\end{array}$ & & $\begin{array}{l}\text { Conversion au } \\
\text { franc }\end{array}$ & & $\begin{array}{l}\text { Livres et } \\
\text { portrait de } \\
\text { Simenon } \\
\end{array}$ & $\begin{array}{l}\text { Gueuze } \\
\text { Herve }\end{array}$ \\
\hline R.I.F. & $\begin{array}{l}\text { La Libre } \\
\text { Belgique }\end{array}$ & $\begin{array}{l}\text { Conversion au } \\
\text { franc }\end{array}$ & Congo & $\begin{array}{l}\text { Portrait } \\
\text { des }\end{array}$ & \\
\hline
\end{tabular}




\begin{tabular}{|l|l|l|l|l|l|}
\hline & & $\begin{array}{l}\text { Nonante/quat } \\
\text { re-vingt-dix }\end{array}$ & $\begin{array}{l}\text { Souverains } \\
\text { (deux fois) } \\
\text { Histoire } \\
\text { belge }\end{array}$ & \\
\hline $\begin{array}{l}\text { Sauveur } \\
\text { Giordano }\end{array}$ & $\begin{array}{l}\text { Fabienne } \\
\text { Vande } \\
\text { Merssche } \\
\text { Le Soir }\end{array}$ & & & & Carapils \\
& & & & \\
\hline
\end{tabular}

\section{b) Les langues nationales}

\section{Le décor sonore}

Les éléments langagiers peuvent être rangés dans trois catégories: l'utilisation de deux des trois langues nationales, l'apparition d'accents et les belgicismes ( $c f$. tableau 9). Le premier constat qu'on peut établir est que la plupart des jeux sur le langage sont utilisés dans une fonction de décor. C'est-à-dire qu'il s'agit d'un fond sonore, d'une particularité d'un personnage (très) secondaire, de détails exotiques qui parsèment les conversations, etc.

Les personnages secondaires sont principalement les porteurs de ces particularismes locaux. Étant donné qu'il a déjà été établi que les personnages principaux sont surtout français, cela parait logique puisque les Belges sont cantonnés aux rôles de second plan. Les personnages secondaires parlent flamand, français avec des accents flamands ou wallon, utilisent des belgicismes, etc.

Il est intéressant de remarquer que le stéréotype de l'accent belge n'est pas aussi présent qu'on aurait pu l'attendre. Il est particulièrement utilisé dans Nestor Burma, et Renseignements généraux, soit deux séries parmi les plus anciennes du corpus. Mais quand il est convoqué, c'est de manière très disqualifiante. En effet, les personnages qui ont un accent prononcé sont idiots. Il s'agit des policiers belges peu compétents de Nestor Burma ou des agents corrompus présents dans Renseignements généraux. La corrélation entre l'accent belge caricatural et le degré de ridicule du personnage peut être perçue comme un rappel du stéréotype présent dans les sketches (ceux de Coluche par exemple) ou les blagues belges.

Les personnages principaux, et donc les Français, s'illustrent parfois dans les jeux de langues. De manière traditionnelle, il s'agit d'abord de constater que les Français prononcent mal les mots flamands, principalement les noms propres (le commissaire Schuiten, le quartier de Matonge...). La plupart du temps, un Belge les reprend et on passe à la scène suivante. Louis Roman fait plus d'efforts. Quand l'épisode 
commence, il est présent depuis quelque temps à Bruges pour participer à un concours de copie de tableau et il semble s'être particulièrement bien adapté à la région. En effet, non seulement il semble comprendre le flamand, mais en plus il le pratique pour des conversations simples. Maryvonne, son épouse qui arrive après lui, ne va pas jusque là, mais elle avoue quand même rêver en flamand depuis qu'elle est en Belgique.

Tableau 9: L'utilisation des particularismes langagiers dans le décor sonore

\begin{tabular}{|c|c|c|}
\hline Néerlandais/français & Accents & Belgicismes/expressions \\
\hline $\begin{array}{l}\text { Annonce des trains dans } \\
\text { les deux langues (L'instit, } \\
\text { Quai n 1) } \\
\text { Les cartes de policiers sont } \\
\text { dans les deux langues } \\
\text { (Renseignements } \\
\text { généraux) } \\
\text { Certaines plaques routières } \\
\text { sont dans les deux langues } \\
\text { (Renseignements } \\
\text { généraux) } \\
\text { Des personnages } \\
\text { secondaires parlent } \\
\text { flamand (Quai no } 1 \text {, Louis } \\
\text { la Brocante) } \\
\text { Des personnages } \\
\text { mélangent les deux langues } \\
\text { (Quai n 1) } \\
\text { Des personnages parlent } \\
\text { français avec un accent } \\
\text { flamand (Louis la } \\
\text { brocante, Le devenir de } \\
\text { l'homme) } \\
\text { Dank U (Louis la } \\
\text { brocante) } \\
\text { Maryvonne rêve en } \\
\text { flamand (Louis la } \\
\text { brocante) } \\
\text { Le steward de l'hôtel } \\
\text { refuse de parler français } \\
\text { (Louis la brocante) } \\
\text { Un dessin animé est en } \\
\text { flamand (R.I.F.) }\end{array}$ & $\begin{array}{l}\text { Des personnages } \\
\text { secondaires ont l'accent } \\
\text { "belge" (Nestor Burma, } \\
\text { Renseignements généraux, } \\
\text { R.I.F.) } \\
\text { Le patron de la casse a un } \\
\text { accent wallon (Sauveur } \\
\text { Giordano) } \\
\text { Nestor Burma prononce } \\
\text { mal le nom du } \\
\text { commissaire (Schuiten) } \\
\text { Un personnage } \\
\text { prononce mal le nom du } \\
\text { quartier Matonge } \\
\text { (Baldi) }\end{array}$ & $\begin{array}{l}\text { Expressions belges } \\
\text { «En Belgique, on dit va te } \\
\text { faire foutre » (Joséphine ange } \\
\text { gardien) } \\
\text { « Non peut-être » } \\
\text { (Renseignements généraux) } \\
\text { Belgicisme } \\
\text { Braderie (L'instit) } \\
\text { Souper (Quai n }{ }^{\circ} \text { 1) } \\
\text { T.D. = thé dansant = une } \\
\text { fête étudiante (Baldi) } \\
\text { Une petite mousse (Baldi) } \\
\text { Tartines (Baldi) } \\
\text { Belgicismes qui sont traduits } \\
\text { par un personnage } \\
\text { Steak tartare/filet américain } \\
\text { (Crimes en séries) } \\
\text { Bourgmestre/maire (A dix } \\
\text { minutes des naturistes, Le } \\
\text { devenir de l'homme) } \\
\text { ONEM/ANPE (Sauveur } \\
\text { Giordano) } \\
\text { Nonante/quatre-vingt-dix } \\
\text { (R.I.F., Le devenir de } \\
\text { l'homme) } \\
\text { Patois bruxellois } \\
\text { Brol (Crimes en séries) } \\
\text { Ket (R.I.F.) }\end{array}$ \\
\hline
\end{tabular}

En gras, l'utilisation des particularismes langagiers utilisés par les héros français

\section{L'incidence des langues sur les intrigues}

Dans une seule série, Nestor Burma, le langage a une incidence sur l'intrigue. Par deux fois, le manque de compétence en langue flamande du commissaire Faroux lui joue des tours. Dans une première scène, il doit 
remettre un prisonnier à des policiers flamands. Les agents qui se présentent sont des usurpateurs, mais il ne se rend pas compte de la supercherie, car leurs cartes professionnelles sont en flamand. Dans une autre scène, il se retrouve dans une situation délicate et appelle le commissaire Schuiten à la rescousse. Comme il est incapable de prononcer son nom convenablement, l'agent à l'accueil du commissariat lui raccroche au nez. Dans les deux cas, la mauvaise compréhension ou utilisation de la langue provoque un rebondissement. Cela a également pour conséquence de ridiculiser le commissaire Faroux. Dans la série, il est traditionnellement présenté comme moins compétent que Nestor Burma et il est régulièrement la victime de situations comiques.

Tableau 10 : L'utilisation des particularismes langagiers dans l'intrigue

\begin{tabular}{|l|l|l|}
\hline \multicolumn{1}{|c|}{ Néerlandais/français } & \multicolumn{1}{|c|}{ Accents } & Belgicismes/expressions \\
\hline Les fausses cartes de & Le commissaire Faroux & \\
policier sont en flamand & prononce tellement mal & \\
(Nestor Burma) & le nom du commissaire & \\
& qu'il ne parvient pas à le & \\
contacter alors qu'il est & \\
dans une situation & \\
& compliquée (Nestor & \\
& Burma) & \\
\hline
\end{tabular}

En gras, l'utilisation des particularismes langagiers utilisés par les héros français

\section{c) Les problèmes linguistiques}

La Belgique est un pays constitué de plusieurs régions (géographiques), communautés (culturelles) et régimes linguistiques. Les problèmes institutionnels ont été rendus célèbres à l'étranger par la crise politique qui a laissé le pays sans gouvernement durant 541 jours du 13 juin 2010 au 6 décembre 2011. Les conflits linguistiques qui opposent les Belges francophones et néerlandophones émaillent également la vie de la nation belge. Ces éléments sont très complexes et nécessitent souvent de longues explications. A priori, ils ne devraient pas se retrouver dans une fiction télévisuelle. Cependant, trois séries du corpus abordent ces problèmes.

Les deux premiers cas sont des conversations où les Belges expliquent la situation au héros français. Goupil (Renseignements généraux) et son homologue belge abordent les problèmes communautaires en buvant une bière dans un bar d'Anvers. L'inspecteur conclut qu'avec les problèmes entre les Wallons et les Flamands, il n'est pas sûr que l'État pour lequel il travaille existera encore dans dix ans. Goupil lui fait remarquer qu'il est bruxellois et donc neutre. Vandenberghe répond que, quand les deux communautés se disputent, «ce sont les Bruxellois qui trinquent». Dans 
Quai $n^{\circ}$ 1, Marie Saint George discute de la région avec Michel Fournier. Ils évoquent les corons flamands, le mélange de cultures, la coexistence des Flamands, des Francophones et des Français (ils sont à quelques kilomètres de la frontière et de Lille). Michel est francophone et son épouse est flamande. Rapidement, la conversation dévie sur le trafic des hormones, mais l'essentiel a été dit sur le multilinguisme ambiant.

Dans Louis la brocante, les personnages principaux sont eux-mêmes victimes des conflits linguistiques. Quand Maryvonne arrive à Bruges, elle s'adresse au steward de l'hôtel qui lui répond en flamand. Évidemment, elle ne comprend pas. Louis arrive et lui explique qu'il s'agit d'un «irréductible. Il ne peut piffer ni les francophones ni les Wallons». Il lui conseille de s'adresser à la patronne de la pension de famille. Un peu plus tard, une amie flamande de Maryvonne lui explique que son beau-fils est francophone, mais que «c'est quand même un bon mari ».

L'épisode de Renseignements généraux date de 1994, celui de Quai n 1 de 1997, celui de Louis la Brocante de 2011. La première conversation a peu d'incidence dans l'histoire et elle est vite oubliée. Le deuxième dialogue est plus important, car le multiculturalisme imprègne le récit de Quai $n^{\circ} 1$. D'ailleurs, le flamand est régulièrement entendu dans l'épisode dans le décor sonore ou dans des conversations entre des personnages secondaires. Les conflits linguistiques sont plus prégnants dans Louis la Brocante où les personnages français les subissent. La chronologie n'est pas sans effet donc. Plus les problèmes linguistiques s'exacerbent dans la réalité, plus ils sont connus à l'étranger, plus ils apparaissent dans la fiction alors que ce ne sont a priori pas des éléments faciles à traiter ni très glamour.

\section{Conclusion}

Les représentations sur les Belges sont visibles dans le corpus, c'est inévitable et nous ne nous attendions pas vraiment à infirmer notre première hypothèse. Cependant les stéréotypes ne sont peut-être pas aussi ostentatoires qu'on aurait pu le croire. En effet, on ne trouve pas de lieu ou de personnage radicalement connoté, on ne décèle pas non plus énormément de clichés dans les épisodes. Ceci peut être interprété de deux façons contradictoires. On peut le regretter en soulignant que les séries ne laissent qu'une place artificielle aux éléments belges. On peut s'en réjouir non pas en affirmant que les fictions étudiées sont exemptes de clichés, ce serait erroné -, mais en remarquant que la distance entre Belges et Français est vraisemblablement trop ténue pour donner lieu à des représentations très discriminantes. 
Une autre explication serait que les représentations véhiculées par les feuilletons sont tellement évidentes (Jodelet, Flahaut) que nous ne les avons pas repérées. Si les réalités belges et françaises sont aussi proches que nous venons de l'affirmer, il se pourrait que nous n'ayons pas été perturbée par les récits visionnés (cf. l'hypothèse d'Isabelle Veyrat-Masson selon laquelle l'on rejette généralement les stéréotypes sur soi énoncés par autrui) ou que nous avons nous-même intégré ces clichés et que nous nous y conformons (autre thèse d'Isabelle Veyrat-Masson discutée précédemment). Ceci démontre en tout cas, la difficulté de travailler sur les représentations sociales, tellement consensuelles qu'elles en deviennent peu visibles. Peut-être qu'un auteur non-belge aurait trouvé d'autres conclusions?

Le corpus est composé d'épisodes de séries françaises coproduits exceptionnellement avec la RTBF. Jean-Pierre Esquenazi souligne que les programmes de télévision sont des "produits" du système qui les crée et qui influence les représentations véhiculées. La nature de ces fictions a plusieurs incidences sur le contenu. D'abord, ces séries sont conçues sur base d'une matrice récurrente comprenant notamment un héros bien identifié. Ce personnage principal et la structure de l'épisode ne changent pas lors de cette coproduction. Les Belges sont donc cantonnés à des rôles secondaires plus ou moins développés. Ensuite, l'escapade en Belgique est exceptionnelle y compris pour les héros voyageurs comme Victor Novak ou Joséphine qui généralement restent au sein de l'Hexagone. Cela peut expliquer certains changements, par exemple, le fait que les récits se déroulent en ville et non dans les milieux ruraux. Cela justifie également que certains éléments typiquement belges soient mis en évidence afin que le téléspectateur comprenne qu'il s'agit d'une aventure inhabituelle. Enfin, les coproductions peuvent s'organiser très différemment en fonction des cas. Les équipes françaises peuvent garder le contrôle de la production du début à la fin, le scénario (tout ou en partie) peut être écrit en Belgique, la réalisation peut être assurée par du personnel belge, etc. C'est un aspect que nous n'avons pas vérifié. Cependant, les résultats de l'analyse de contenu montrent que les intriguent ne s'inscrivent que superficiellement dans le contexte belge. Soit la production a été prise en charge par des Français (les épisodes sont alors des "discours", selon Jean-Pierre Esquenazi, de Français sur les Belges), soit les auteurs belges se sont inscrits dans le cercle vicieux décrit par Isabelle Veyrat-Masson et ont reproduit les comportements qu'on attendait d'eux.

Dans tous les cas, ces coproductions véhiculent des stéréotypes sur la Belgique et les Belges. À l'issue de ce travail d'analyse, quelques mécanismes de représentation peuvent être soulignés.

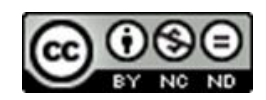


Tout d'abord, il est évident que les représentations sont schématiques. La Belgique présentée est urbaine, bruxelloise et se résume à quelques quartiers identifiables ou quelques monuments. Comme le notait Céline Bryon-Portet, cela participe de l'effet de réel puisque ces images ont pour fonction de déterminer le lieu de l'action. D'où l'importance des séquences de déplacement qui annoncent le voyage du personnage principal. En même temps, et c'était également une conclusion de Céline Bryon-Porter, cela réduit la Belgique à sa capitale. Les images de la Belgique servent de décor aux intrigues, mais ne plongent pas véritablement les personnages dans un univers belge.

Céline Bryon-Portet, en citant les références par exemple à la gastronomie marseillaise repérée dans Plus belle la vie, remarquait que seuls les traits les plus populaires sont repris. C'est également le cas, des particularismes belges. L'examen montre que des éléments issus des médias, de la culture, de la gastronomie parsèment les intrigues. De nouveau, ces détails font partie du contexte et n'impactent que rarement les scénarios. On y retrouve des stéréotypes notoires sur la Belgique : la bière, Saint Nicolas, l'utilisation du "nonante", etc. Globalement, l'image de la Belgique qui émerge de ces épisodes est relativement homogène et stable confirmant que les stéréotypes nationaux reposent sur des «traits de caractère communs et immuables » (Crouzet 134).

Si cette tendance à la schématisation est lourde, cela ne signifie pas qu'aucune séquence n'est à retenir. D'autres lieux sont illustrés parfois. Sauveur Giordano nous emmène à Nivelles, Renseignements généraux à Anvers et Louvain-la-Neuve, Quai $n^{\circ} 1$ dans la campagne flamande. L'analyse montre également que le flamand et les particularismes langagiers sont présents dans le décor sonore de beaucoup d'épisodes. Certaines séries (Renseignements généraux, Quai $n^{\circ} 1$ et Louis la Brocante) parviennent même à aborder les conflits linguistiques qui divisent le pays. C'est un point à souligner, car ces faits sont très compliqués et conviennent assez peu au format série a priori.

Les propos de Sylvaine Marandon trouvent également confirmation dans notre corpus. Selon elle, les stéréotypes servent à qualifier des personnages secondaires pittoresques. Dans nos coproductions, ce sont effectivement les personnages très secondaires qui sont les plus clichés. Ce sont par exemple les seuls à posséder le fameux accent belge. Ce trait rendu célèbre par les blagues sur les Belges ou les sketches d'humoristes est relativement peu présent dans les fictions étudiées et se cantonne aux plus anciennes. Mais il est difficile d'affirmer que les Belges se réduisent à des personnages rigolos. En effet, nous l'avons abordé dans le point sur la forme de la coproduction, les épisodes doivent se couler dans le format habituel. Le héros français est incontournable et, parfois, ses acolytes. Il est 
dès lors compliqué de prévoir ce que les représentations des Belges auraient donné si les personnages avaient eu l'occasion d'accéder aux places principales. Il faut cependant noter qu'on n'assiste pas à une répartition entre les "bons" Français et les "méchants" Belges, en tout cas dans la plupart des séries.

Une évolution est perceptible au fil des années. Nous l'avons déjà précisé, les séries utilisant l'accent belge sont les plus anciennes. Dans les productions contemporaines, il a disparu. Mais le trait le plus évident à ce propos est l'utilisation de plus en plus importante des langues nationales en décor sonore, dans les dialogues et, pour trois séries, dans les thématiques. Si le conflit linguistique n'était évoqué que rapidement au détour d'une conversation dans Renseignements généraux (1994), cela imprégnait le contexte de l'intrigue dans Quai $n^{\circ} 1$ (1997) et avait un impact sur les personnages principaux dans Louis la brocante (2011). Il est évidemment que les scénaristes ont abandonné la vision simpliste d'un pays où les habitants ont des accents bizarres pour comprendre le fond culturel et politique que cela suppose. On pourrait également conclure qu'internationalement, l'image de la Belgique est de plus en plus impactée par ses conflits linguistiques au point que, malgré la complexité, ils deviennent incontournables pour les scénaristes.

Il faut pourtant se garder de penser que les clichés sur les Belges ont disparu des séries. Le cas des personnages très secondaires disqualifiés est à ce propos éloquent. En effet, on en retrouve autant dans les anciennes séries que dans les nouvelles. Dans R.I.F., qui date de 1995, un scénario manichéen oppose des malfrats corses et belges opposés à de vaillants policiers français. Dans Plus belle la vie (2010), les héros français se heurtent à des figures très convenues de policiers stupides et corrompus. Ceci confirme, de surcroît, la proposition de François Crouzet qui indique que plusieurs strates de stéréotypes peuvent exister. En effet, au moment où Plus belle la vie utilise un procédé réducteur, Louis Roman (Louis la brocante) parle flamand.

Les stéréotypes nationaux sont révélateurs des relations entre les pays, mais aussi de l'identité de la nation qui produit le discours, avons-nous indiqué dans la partie théorique de cet article. Que nous disent les clichés répertoriés de la relation entre la Belgique et la France ? Le dialogue issu de R.I.F. déjà cité nous semble bien symboliser les analyses. La Belgique n'est pas exotique pour les Français (moins que la Guyane avons-nous écrit plus haut), mais offre malgré tout un sentiment de décalage. Amener les personnages dans le Plat pays offre à la fois un univers connu tant les deux cultures sont proches et un petit dépaysement. Il n'est dès lors pas étonnant que les stéréotypes soient peu nombreux et de l'ordre du détail.

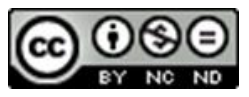


Cependant, les épisodes étudiés présentent une Belgique "prête à consommer" pour un public français. Ce sont certaines bizarreries qui le laissent penser. D'abord, des personnages belges prononcent le nom "Bruxelles" à la française avec un "X" et non un "S". Ensuite, la focalisation sur l'Atomium ne peut que paraitre artificielle pour un Belge, alors que cela doit être très lisible pour un Français habitué à voir la tour Eiffel dans toutes les images sur l'Hexagone. Enfin, la mise en évidence de Bruxelles en dit long sur l'omniprésence de Paris dans l'imaginaire français.

Isabelle Veyrat-Masson remarquait que la représentation des États-Unis était étonnement riche dans le corpus qu'elle a examiné. Elle soulignait que les stéréotypes ne sont pas absents des séries américaines car ils sont incontournables, mais que leur multiplication enrichit les images sur le pays. Elle ajoutait que, contrairement aux autres nations, les États-Unis contrôlent leur propre représentation, car ils produisent eux-mêmes les séries. Ce n'est pas le cas de la Belgique. Les voyages de héros français dans le plat pays restent exceptionnels et les représentations des Belges sont quantitativement peu importantes et donc, qualitativement, relativement pauvres. Les stéréotypes répertoriés ici sont l'objet des Français et non des productions belges. La solution réside probablement dans une production nationale de fiction plus soutenue. 


\section{Bibliographie}

Amossy, Ruth. Les idées reçes. Sémiologie du stéréotype. Paris : Nathan, 1991.

Anonyme. "21 préjugés sur les Belges.” Femmes d'aujourd'hui 292013 : 1217.

Anonyme. "Chronologie de l'affaire." tempsreel.nouvelobs.com. 22 juin 2004. Web. 30 Aug. 2015.

Anonyme. "Stéréotypes européens." Europe is not dead, 2012. Web. 30 Aug. 2015.

Benassi, Stéphane. Séries et feuilletons T.V. Pour une typologie des genres fictionnels télévisuels. Liège: Éditions du Cefal, 2000.

Blandin, Claire. "Les Maittres de l'orge. 'Une sage de l'écrit à l'écran'." Le temps des médias 14 (2010/1): 142-157. Web. 30 Aug. 2015.

Boyer, Henri. "Stéréotype, emblèmes, mythe. Sémiotisation médiatique et figement représentationnel." Mots. Les langages du politique 88 (2008): n. pag. Web. 15 Oct. 2010.

Bryon-Portet, Céline. "Les productions télévisées, genre oublié dans la construction de l'image d'un territoire? L'exemple de coconstruction de l'image socioculturelle de la ville de Marseille par la série Plus belle la vie." Études de communication 37 (2011): 79-96. Web. 30 Aug. 2015.

Bryon-Portet, Céline. "La dimension politique de la série Plus belle la vie. Mixophilie, problématiques citoyennes et débats socioculturels dans une production télévisuelle de service public." Mots. Les langages du politique 99 (juillet 2012): 97-112. Web. 30 Aug. 2015.

Chalvon-Demersay, Sabine. Mille scénarios, une enquête sur l'imagination en temps de crise. Paris: Édition Métailié, 1994.

Chalvon-Demersay, Sabine. "La confusion des conditions. Une enquête sur la série télévisée Urgences." Réseaux 95 (1999): 237-281. Web. 30 Aug. 2015.

Chalvon-Demersay, Sabine. "Le second souffle des adaptations." L'Homme 175-176 (2005/3): 77-111. Web. 30 Aug. 2015.

Crouzet, François. "Images d'outre-Manche: la France vue par les Britanniques, la Grande-Bretagne vue par les Français, 1904-2004.” Histoire, économie et société 1 (2006): 131-141. Web. 30 Aug. 2015.

Dagnaud, Monique. Les artisans de l'imaginaire. Comment la télévision fabrique la culture de masse. Paris: Armand Colin, 2006.

Dufays, Jean-Louis. Stéréotype et lecture. Liège: Mardaga, 1994.

Eco, Umberto. "James Bond : une combinatoire narrative." Communications 8 (1966): 77-93. Web. 30 Aug. 2015.

Ephimenco, Sylvain. "Un vétérinaire belge, Karel van Noppen, victime de la 'mafia des hormones"." Libération. 28 février 1995. Web. 30 Aug. 2015. 
Esquenazi, Jean-Pierre. "Elements de sociologie sémiotique de la télévision." Quaderni 50-51 (printemps 2003): 89-115. Web. 30 Aug. 2015.

Flahaut, François. "Récits de fiction et représentations partagées." L'bomme 175-176 (2005/3): 37-55. Web. 30 Aug. 2015.

Glesener, Thomas. "Flandre et Flamands dans l'imaginaire espagnol du XVIe siècle." Revue du Nord 360- 361 (2005/2): 337-350. Web. 30 Aug. 2015.

Heinich, Nathalie. "Les limites de la fiction." L'bomme 175-176 (2005/3): 57-76. Web. 30 Aug. 2015.

Jodelet, Denise. "Représentations sociales: phénomènes, concept et théorie." Psychologie sociale. Éd. Serge Moscovici. Paris: Presses universitaires de France, 2003. 363-384.

Lafon, Benoit. 'Des fictions 'toutes proches': une certaine identité de la France. Enjeux politiques des séries télévisées de France 3 en prime time (Louis la Brocante, Famille d'accueil, Un Village français)." Mots. Les langages du politique 99 (juillet 2012): 79-95. Web. 30 Aug. 2015.

Macé, Éric. La société et son double: une journée ordinaire de télévision française. Paris: Armand Colin, 2006.

Marandon, Sylvaine. "Stéréotypes nationaux et préjugés raciaux du XIXe siècle. Sources et méthodes à travers l'exemple anglais." Stéréotypes nationaux et préjugés raciaux aux XIXe et XXe siècles. . Sources et méthodes pour une approche historique. Éd. Jean Pirotte. Louvain-la-Neuve: Naumelaerts, Collège Érasme, Bureau du Recueil, 1982. 3-18.

Moscovici, Serge. "Des représentations collectives aux représentations sociales : éléments pour une histoire.", Les représentations sociales. Éd. Denise Jodelet. Paris: Presses universitaires de France, 1989. 62-86.

Pasquier, Dominique. La culture des sentiments. L'expérience télévisuelle des adolescents. Paris : Éditions de la Maison des Sciences de l'Homme, 1999.

Pirotte, Jean, ed. Stéréotypes nationaux et préjugés raciaux aux XIXe et XXe siècles. Sources et méthodes pour une approche historique. Louvain-laNeuve : Naumelaerts, Collège Érasme, Bureau du Recueil, 1982.

Sépulchre, Sarah. "Melting Pot: An ambiguous series combining minority and majority discourses." Alternative francophone 1. 6 (2013): n. pag. Web. 30 Aug. 2015.

Tilleux-Craenhals, Ghislaine. "L'autostéréotype du Belge dans la bande dessinée." Stéréotypes nationaux et préjugés raciaux aux XIXe et XXe siècles. . Sources et méthodes pour une approche historique. Éd. Jean Pirotte. Louvain-la-Neuve: Naumelaerts, Collège Érasme, Bureau du Recueil, 1982. 117-133.

Veyrat-Masson, Isabelle. "Les stéréotypes nationaux et le rôle de la télévision (1).” Hermès 5-6 (1989): 237-253. Web. 30 Aug. 2015.

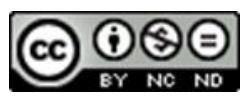

